\title{
NUMERICAL COMPUTATION OF SOLITONS FOR OPTICAL SYSTEMS
}

\author{
LAURENT Di MenzA ${ }^{1}$
}

\begin{abstract}
In this paper, we present numerical methods for the determination of solitons, that consist in spatially localized stationary states of nonlinear scalar equations or coupled systems arising in nonlinear optics. We first use the well-known shooting method in order to find excited states (characterized by the number $k$ of nodes) for the classical nonlinear Schrödinger equation. Asymptotics can then be derived in the limits of either large $k$ are large nonlinear exponents $\sigma$. In a second part, we compute solitons for a nonlinear system governing the propagation of two coupled waves in a quadratic media in any spatial dimension, starting from one-dimensional states obtained with a shooting method and considering the dimension as a continuation parameter. Finally, we investigate the case of three wave mixing, for which the shooting method is not relevant.
\end{abstract}

Mathematics Subject Classification. 35J25, 35J60, 65N06, 65N99, 78M20.

Received July 14, 2006. Revised April 7, 2008.

Published online December 5, 2008.

\section{INTRODUCTION}

Solitons have always been considered as a object of fascination in optics, as spatial structures propagating in nonlinear media with shape invariance. Among all the possible models investigated for the description of wave propagation, a well-known equation relevant in nonlinear optics as well as plasma physics or quantum mechanics is the nonlinear Schrödinger equation (NLS)

$$
i \frac{\partial \psi}{\partial t}+\Delta \psi+\alpha|\psi|^{2 \sigma} \psi=0, \psi=\psi(t, x)
$$

where $x \in \mathbb{R}^{d}$ is the transverse coordinate, $t \in \mathbb{R}^{+}$is the propagation distance normalized to the diffraction length, $\alpha \in \mathbb{R}, \sigma>0$ and $\Delta=\partial^{2} / \partial x_{1}^{2}+\ldots+\partial^{2} / \partial x_{d}^{2}$ (one can refer to [17] for the derivation of (1.1) in various physical contexts). Note that the specific case $\sigma=1$ leads to the cubic equation that corresponds to wave propagation in Kerr media: the nonlinear index is then proportional to the field intensity $|\psi|^{2}$. Seeking for solutions on the form $\psi(t, x)=u(x) \exp (i \omega t)$, where $\omega$ is real and $u$ is assumed to be localized in space (say, a solution that fastly decreases to zero as $r:=\|x\|$ goes to infinity; this kind of solution can be denoted by a bright soliton) equation (1.1) turns into the nonlinear elliptic problem

$$
-\omega u+\Delta u+\alpha|u|^{2 \sigma} u=0, x \in \mathbb{R}^{d} .
$$

\footnotetext{
Keywords and phrases. Nonlinear optics, elliptic problems, stationary states, shooting method, continuation method.

1 Université de Reims, Laboratoire de Mathématiques, Moulin de la Housse, BP 1039, 51687 Reims Cedex 2, France.

laurent.di-menza@univ-reims.fr
} 
In the one-dimensional case $d=1$, such an equation can be explicitly integrated for $\omega>0$ and $\alpha>0$ to find sech-profiles and only nonnegative localized states can be obtained. For higher space dimensions, the situation becomes completely different: for the same assumptions made on $\omega$ and $\alpha,(1.2)$ admits many localized radial solutions for reasonable values of $\sigma$ (namely, $\sigma>0$ if $d \leq 2$ and $0<\sigma<\sigma^{*}=2 /(d-2)$ if $d \geq 3$ ) that can be parameterized by the number $k$ of nodes. These solutions $u_{k}$ are often referred as bound states or excited states, for the sake of correspondence with quantum mechanics formalism; see for example [13]. The localized solutions of (1.2) can be seen as critical points of the action $S(u)$ defined as

$$
S(u):=\frac{\omega}{2} \int_{\mathbb{R}^{d}}|u(x)|^{2} \mathrm{~d} x+\frac{1}{2} \int_{\mathbb{R}^{d}}\left(\|\nabla u(x)\|^{2}-\frac{\alpha}{\sigma+1}|u(x)|^{2(\sigma+1)}\right) \mathrm{d} x
$$

and a variational framework enables to show existence of such solutions [17]. A vast literature can be found concerning stationary states of these equations, with particular attention paid on the connection between the so-called ground state $u_{0}$ defined as the only strictly positive solution in $H^{1}\left(\mathbb{R}^{d}\right)$ of (1.2) [10] and blow-up occurrence of the solution of (1.1) in the focusing critical case $\sigma=2 / d$ : it is well-known (see [19]) that if $\psi_{0} \in H^{1}\left(\mathbb{R}^{d}\right)$ is such that $\left\|\psi_{0}\right\|_{L^{2}\left(\mathbb{R}^{d}\right)}<\left\|u_{0}\right\|_{L^{2}\left(\mathbb{R}^{d}\right)}$, then the solution $\psi$ of (1.1) such that $\psi(0)=\psi_{0}$ is global in time and $\|\psi(t)\|_{H^{1}\left(\mathbb{R}^{d}\right)}$ remains uniformly bounded in time.

Recently, more general models have been investigated for the concern of propagation in media where the susceptibility tensor is quadratic in terms of input fields. A typical example is given by the so-called type I polarization, governed by the system

$$
\left\{\begin{array}{c}
i \frac{\partial u}{\partial z}+\Delta u-u+\bar{u} v=0 \\
i \sigma \frac{\partial v}{\partial z}+\Delta v-\rho v+\frac{1}{2} u^{2}=0
\end{array}\right.
$$

up to several assumptions as well as various rescalings in longitudinal and transverse domains (see [3] for a review of quadratic models in nonlinear optics). This model describes the propagation of the fundamental wave $u$ of frequency $\omega$ and the first harmonic wave $v$ characterized by frequency $2 \omega$. Stationary bright solutions have been obtained in this context with different methods: the first one enabled to compute approximate values of ground states from a Gaussian ansatz, leading to the resolution of a nonlinear algebraic system (see for instance [2]). Shooting method or relaxation technique (starting from explicit solutions that are obtained for particular values of the parameters) have also permitted to exhibit excited states. In [7], stationary states have been topologically classified with use of a Newtonian description. Other studies have been made for spatio-temporal solitons leading to nonradial structures when taking into account time dispersion effects (see $[12,18]$ ).

This paper is organized as follows: in Section 2, we describe the way of computing solitons states for (1.1) using the shooting method and give some numerical illustrations. We also obtain localized solutions in the sublinear case $-1 / 2<\sigma<0$ (for which the nonlinear term still makes sense even if the nonlinear index cannot be defined) that appear to be compactly supported in space, as opposed as what has been found for nonnegative $\sigma$. Moreover, two-dimensional vortex-like solutions are computed with this technique. Finally, the asymptotics $k \rightarrow \infty$ and $\sigma \rightarrow \sigma^{*}$ are numerically investigated, leading us to scaling laws in terms of $k$ or $\sigma^{*}-\sigma$. In Section 3, we investigate the case of system (1.3) starting from one-dimensional bright bound states computed with the shooting method. A continuation technique is then used for the determination of higher space dimension solutions as well as vortex solitons. In the last section, we deal with a model involving three coupled waves propagating in a quadratic media. In this situation, we show that it is not possible to start using the shooting method from the one-dimensional case as it was the case for previous two-wave model. A continuation argument is then applied to carry out solitons in any space dimension. 


\section{SOlitons fOR NLS EQUATION}

We first seek solutions of (1.1) that write $\psi(t, x)=\mathrm{e}^{i \omega t} u(r)$ (with $\omega>0$ and $r=\|x\|$ ), leading us to the elliptic equation (1.2) that will be seen later as a differential second-order equation. This problem has been intensively studied and it has been proved using variational arguments that in the case $\alpha>0$ and $\omega>0$, $H^{1}$ solutions exist only when $\sigma<\sigma^{*}$ (which means for every $\sigma>0$ if $d \leq 2$ ). Note that nonexistence results can be obtained as a consequence of Pohozaev identities (see [17] and references therein). First, a simple rescaling argument shows that it is always possible to assume that $\omega=1$ : if $u$ denotes a solution of (1.2), then for each $\lambda>0$, the new function $\tilde{u}(r)=\lambda^{1 / \sigma} u(\lambda r)$ solves (1.2) with $\tilde{\omega}=\omega \lambda^{2}$. Taking $\lambda=1 / \sqrt{\omega}$ thus leads to the correctly rescaled equation. Furthermore, looking for a $C^{2}$ solution at $r=0$ requires to set $u^{\prime}(0)=0$ in order to avoid a singular point at the origin. We then investigate the differential system which expresses as

$$
\left\{\begin{array}{l}
u^{\prime \prime}(r)+\frac{d-1}{r} u^{\prime}(r)-u(r)+\alpha|u(r)|^{2 \sigma} u(r)=0, \quad r>0 \\
u(0)=\beta, \quad u^{\prime}(0)=0
\end{array}\right.
$$

and we look for globally defined solution of (2.1) that tends to zero as $r \rightarrow \infty$. First, this system can be rewritten as a general first-order system of the form $X^{\prime}(r)=F(r, X(r))$ with $X(r)=\left(u(r), u^{\prime}(r)\right) \in \mathbb{R}^{2}$. Here, the function $F$ may be delicate to evaluate at $r=0$ because of the singular term $u^{\prime}(r) / r$ that arises as a consequence of radial geometry assumption. Indeed, a Taylor expansion around $r=0$ of the derivative leads us to $u^{\prime}(r) / r \sim u^{\prime \prime}(0)$ as $r \rightarrow 0$. Consequently, we have $u^{\prime \prime}(r)+(d-1) u^{\prime}(r) / r \sim d u^{\prime \prime}(0)$ and the second derivative of $u$ at zero can be expressed using (2.1) as $u^{\prime \prime}(0)=\left(\beta-\alpha|\beta|^{2 \sigma} \beta\right) / d$. The initial data $\beta$ should be prescribed in such a way that the corresponding solution has nice decreasing properties at infinity.

\subsection{The shooting method for bright solitons}

We first present the numerical algorithm in order to find the ground state denoted $u_{0}$ of (1.1), that is with no node. This algorithm is based on the fact that (1.2) admits a unique positive radial solution that tends to zero at infinity (see [10]), associated to some initial value $\beta=\beta^{*}$ in (2.1) to be determined. At each step, say $n$, the exact value $\beta^{*}$ is seeked in an interval $\left[a_{n}, b_{n}\right]$. We compute $\beta_{n}=\left(a_{n}+b_{n}\right) / 2$ and we numerically solve (2.1) for $\beta=\beta_{n}$. If the approximate solution becomes negative at some $r_{0}$, then $\beta^{*}<\beta_{n}$ and we set $a_{n+1}=a_{n}$ and $b_{n+1}=\beta_{n}$. If the solution is always larger then some small prescribed value $\varepsilon>0$, then we prescribe $a_{n+1}=\beta_{n}$ and $b_{n+1}=b_{n}$. The initial interval is chosen in such a way that $a_{0}<\beta^{*}<b_{0}$ : $a_{0}$ is set equal to zero and $b_{0}$ is such that the solution of the corresponding Cauchy problem changes its sign.

We give in Figures 1 and 2 an illustration of this method for the determination of the ground state on the numerical domain $[0,10]$ in the focusing cubic bidimensional case $\alpha=\sigma=1$ and $d=2$ (often referred in the literature as the Townes soliton), starting from $b_{0}=4$. In all these experiments, (2.1) has been solved with use of the fourth-order Runge-Kutta method, meaning that approximate solutions of the soliton are computed on prescribed discrete points $r_{j}=j \delta r(0 \leq j \leq N)$ where $N$ is given and $\delta r=R / N$. At the second step, we have $a_{1}=2$ and $b_{1}=4$ and taking $\beta_{1}=\left(a_{1}+b_{1}\right) / 2$ gives us a solution that changes its sign, which indicates that the expected value lies in $\left[a_{1}, \beta_{1}\right]$ (see Fig. 1 ). We thus set $a_{2}=a_{1}$ and $b_{2}=\beta_{1}$. After 10 iterations, the solution calculated from $\beta_{9}=\left(a_{9}+b_{9}\right) / 2$ still changes its sign and consequently, we set $a_{10}=a_{9}$ and $b_{10}=\beta_{9}$ (see Fig. 2). Of course, the number of iterations in this algorithm is closely related to the size of the prescribed computational domain $[0, R]$. We decided to stop our computations as soon as the numerical solution of (2.1) no longer differs within this bounded domain between two consecutive dichotomy iterations. It is observed that the solution that is obtained is in good agreement with the numerical approximation of the ground state that can be found in the literature (see for example [19]) and that is well-known by physicists, using for example Gaussian ansatz and finite-dimensional minimization algorithm to get an approximate profile (this method can be extended to more general models, see [16]). We then found a value at the origin for the Townes soliton given by $\beta^{*} \approx 2.2062027369 \ldots$ 


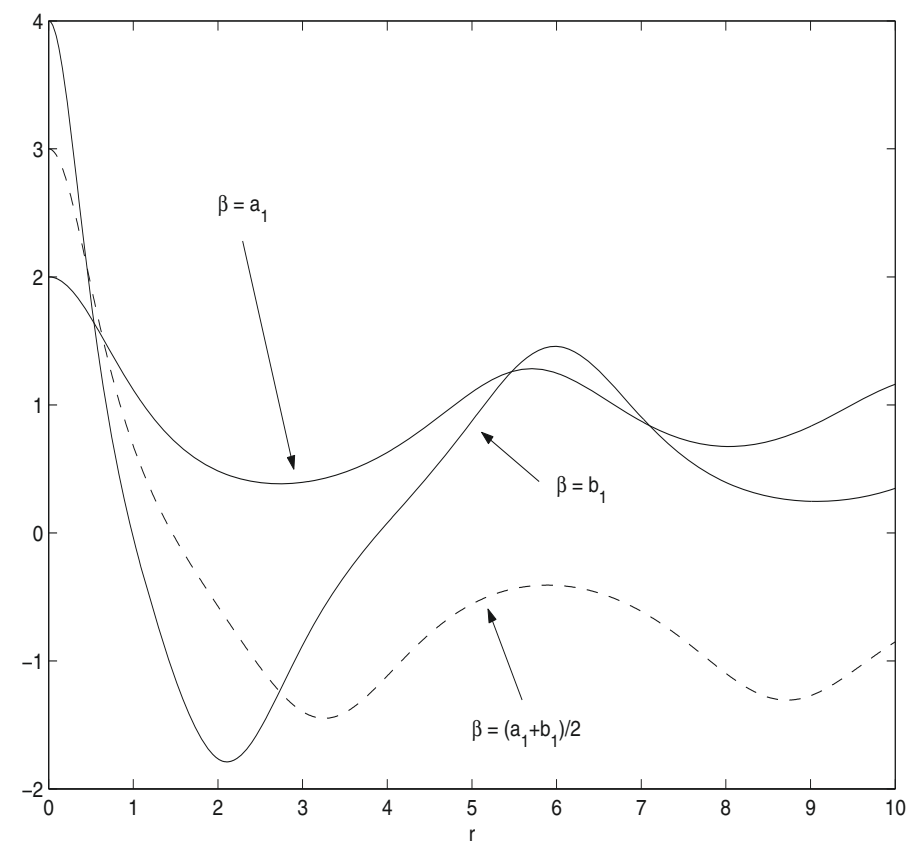

Figure 1. Determination of the ground state: second iteration $\left(a_{1}=2, b_{1}=4\right), d=2$, $\alpha=\sigma=1$.

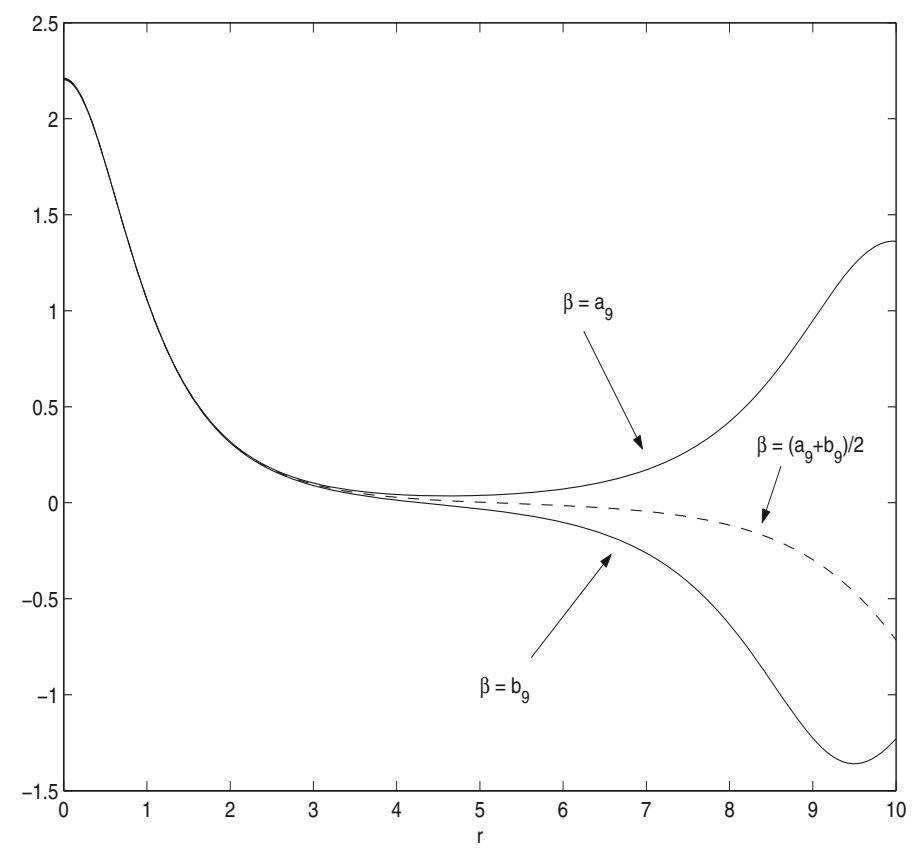

Figure 2. Determination of the ground state: 10th iteration $\left(a_{9}=2.203125, b_{9}=2.2109375\right)$, $d=2, \alpha=\sigma=1$. 


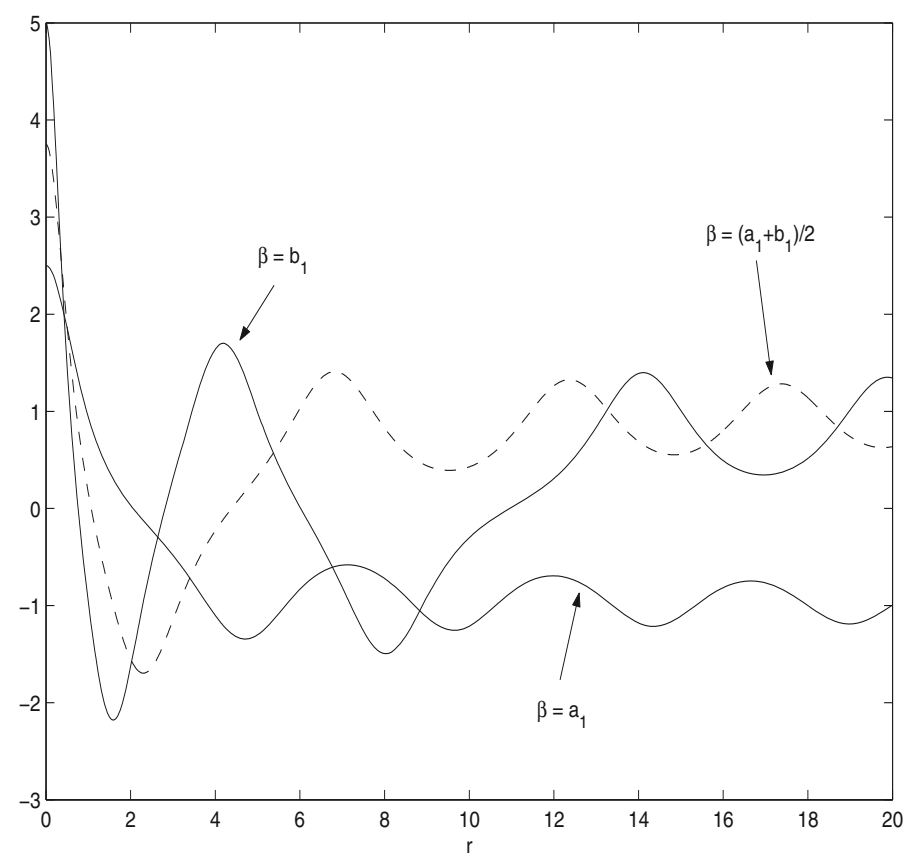

Figure 3. Determination of the 2nd excited state: second iteration $\left(a_{1}=2.5, b_{1}=5\right)$.

The algorithm for the determination of the $k$ th excited state $u_{k}$ is very similar to the one previously described. Here, at step $n$, if the solution computed with $\beta_{n}$ changes its sign more than $k$ times, we fix $a_{n+1}=a_{n}$ and $b_{n+1}=\beta_{n}$. In the opposite case, we prescribe $a_{n+1}=\beta_{n}$ and $b_{n+1}=b_{n}$. We present in Figures 3 and 4 the results obtained for $n=1$ and $n=9$ on the computational domain $[0,20]$ initializing the method with $a_{0}=5$ for the calculation of the second excited state of (1.2) still considered for $\alpha=\sigma=1, d=2$. In this case, it is observed that taking $\beta_{1}=\left(a_{1}+b_{1}\right) / 2$ leads us to a solution that vanishes 2 times on $[0, R]$ but which does not tend to zero at infinity. After a few iterations, the profile of the excited state begins to stabilize for $r \in[0,10]$ (see Fig. 4) and the remaining amount of mass is evacuated at large distances.

In all these computations, the bounded interval $[0, R]$ is such that $R$ is assumed to be larger than all the nodes of the seeked solution. In our experiments, we choose $R$ sufficiently large and check that the computed solution with this algorithm does not depend on $R$. Moreover, the approximate solution of (2.1) depends on the numerical scheme and one has to deal with sufficiently large values of $N$ to grant that the error given with the method is small enough. It has been observed that the value at the origin given by the shooting method converges to a limit value when $N$ large enough. Lastly, the upper bound $b_{0}$ has to be chosen sufficiently large in order to have $u_{k}(0)<b_{0}$. It suffices to take the value at the origin of a solution of (2.1) that vanishes at least $k+1$ times. We have validated this method in the one-dimensional case: we have checked that the solution numerically found perfectly mimics the exact one explicitly given by

$$
u(x)=\frac{(\sigma+1)^{1 / 2 \sigma}}{(\cosh \sigma x)^{1 / \sigma}}, x \in \mathbb{R}
$$

obtained by a direct integration of (1.2). From now on, the value of $u_{k}$ at the origin will be noted $u_{0}^{k}$.

In Figure 5, we plot the results obtained when looking for the ground state $u_{0}$ and the first two excited states $u_{1}$ and $u_{2}$, for the same values of $d, \alpha$ and $\sigma$ as before. We also view in Figure 6 the plot of the logarithm of the solution, noticing that the asymptotics does not depend on the number of nodes. Indeed, in this pure 


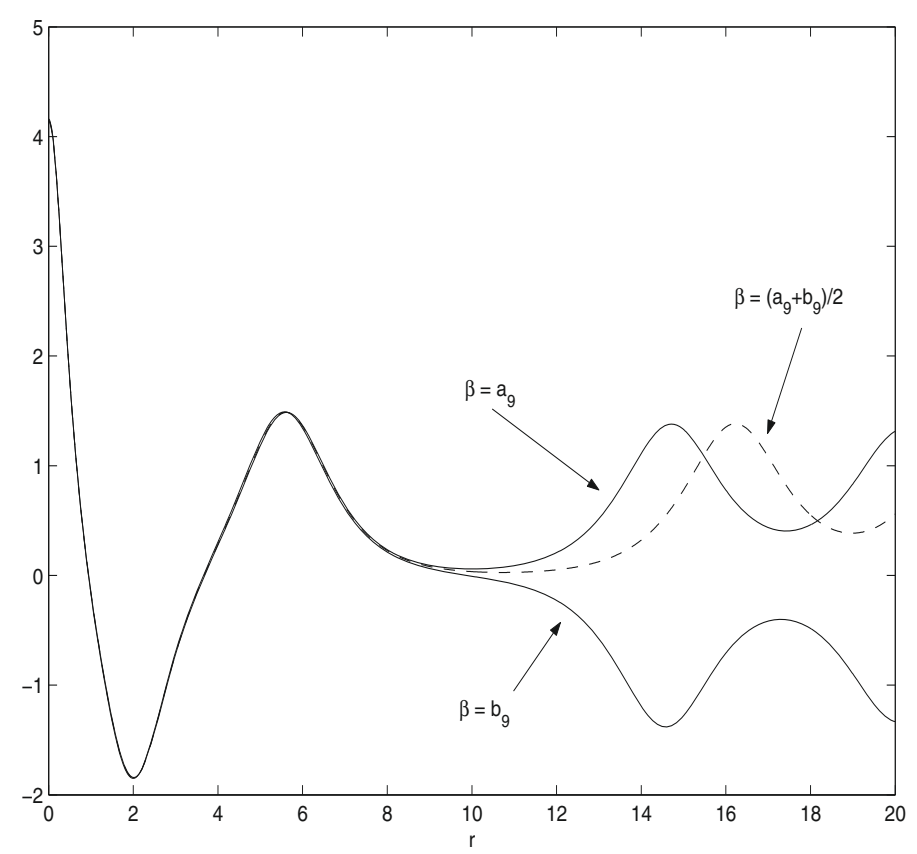

FigurE 4. Determination of the 2nd excited state: 10 th iteration $\left(a_{9}=4.140625, b_{9}=4.150390625\right)$.

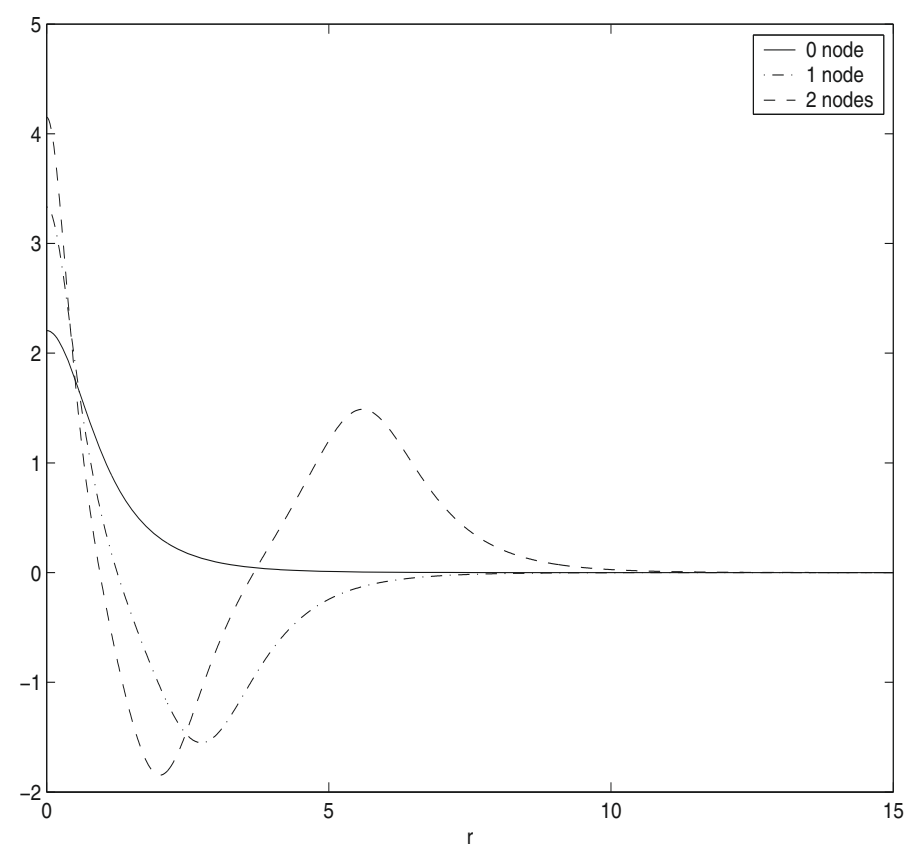

FigurE 5. The first three stationary states of (1.1), $d=2, \alpha=\sigma=1$. 


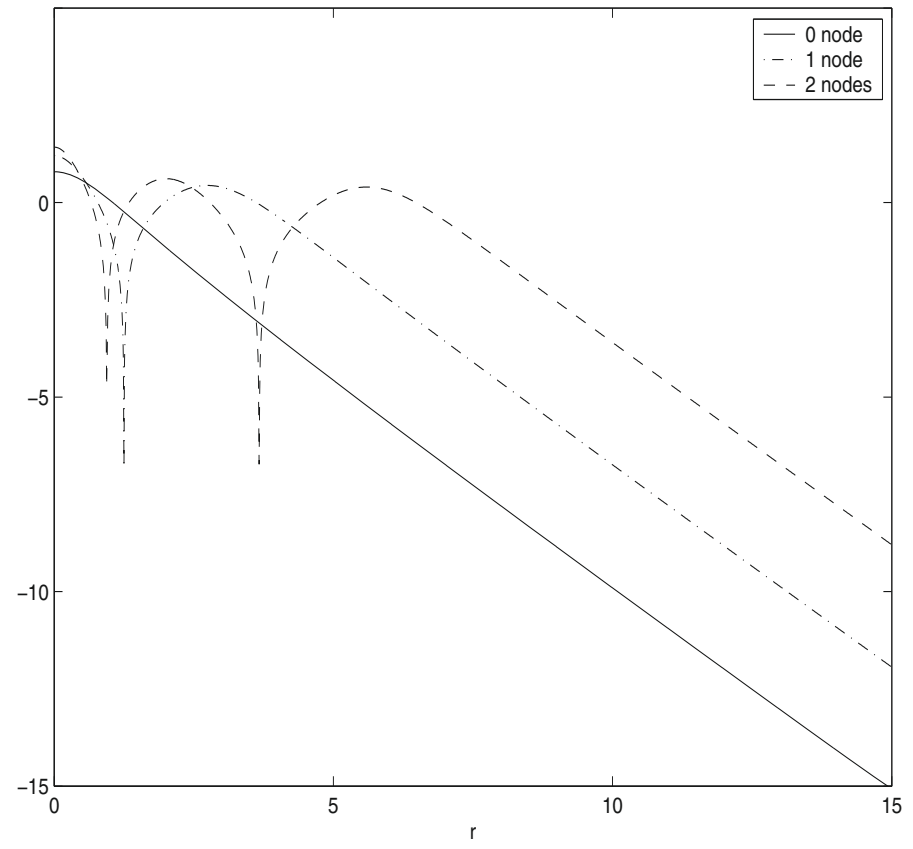

Figure 6 . Plot of the $\log$ of the solutions, $d=2, \alpha=1=\sigma=1$.

power case, the solution behaves similarly to the one of the linear problem that is given by $u(r) \sim C \mathrm{e}^{-r} / r^{(d-1) / 2}$ $(r \rightarrow \infty)$ and the $\log$ profile will exhibit a linear evolution slightly corrected by the perturbation $(d-1) \log r / 2$. As said before, the choice of parameter $R$ depends on the number of nodes of the expected solution: it has been noticed that for a given $\varepsilon>0$, the quantity $R_{k}^{\varepsilon}:=\max \left\{r \geq 0:\left|u_{k}(r)\right| \geq \varepsilon\right\}$ is an increasing sequence. Thus, the $k$ th excited state will become less spatially localized for large $k$. Finally, we have observed that $u_{k}$ possesses $k$ local extrema that satisfy the following property: the first one is attained at the origin and the $k-1$ other ones are located at some $r_{j}>0(j \in\{1, \ldots, k\})$ such that $\forall j \in\{1, \ldots, k\}, u_{0}^{k}>\left|u_{k}\left(r_{j}\right)\right|$ and consequently $\left\|u_{k}\right\|_{L^{\infty}\left(\mathbb{R}^{d}\right)}=u_{0}^{k}$. This statement can be proved using Appendix C in [5].

\subsection{Vortex solitons in the two-dimensional case}

In the particular case $d=2$, it is also possible to look for stationary states that express as $\psi(t, x)=$ $\mathrm{e}^{\mathrm{i} \omega t} \mathrm{e}^{\mathrm{i} m \theta} u(r)$, where $m$ is an integer (sometimes known as the vortex charge or the winding number) and $(r, \theta)$ stand for the polar coordinates of $x$ (that is $\left(x_{1}, x_{2}\right)=(r \cos \theta, r \sin \theta)$ with $\theta \in[0,2 \pi[)$. That means that the spatial solution is no longer radial, even if the function $u$ still depends on $r$. Plugging this expression into (1.1), we now find the new ordinary differential equation

$$
u^{\prime \prime}(r)+\frac{1}{r} u^{\prime}(r)-\frac{m^{2}}{r^{2}} u(r)-u(r)+\alpha|u(r)|^{2 \sigma} u(r)=0, r>0 .
$$

As opposed to the previous situation, seeking a smooth profile requires to prescribe $u(0)=0$ due to the new term $u(r) / r^{2}$ in (2.2) and another shooting parameter has to be found in this case. We then set $u(r)=r^{m} U(r)$ in order to get rid of the term $m^{2} u(r) / r^{2}$. It is easy to check that the new function $U$ solves the modified equation

$$
U^{\prime \prime}(r)+\frac{2 m+1}{r} U^{\prime}(r)-U(r)+\alpha r^{2 m \sigma}|U(r)|^{2 \sigma} U(r)=0, r>0
$$

which closely looks like the one that had to be solved in the radial case, except that the nonlinearity now depends on $r$. 


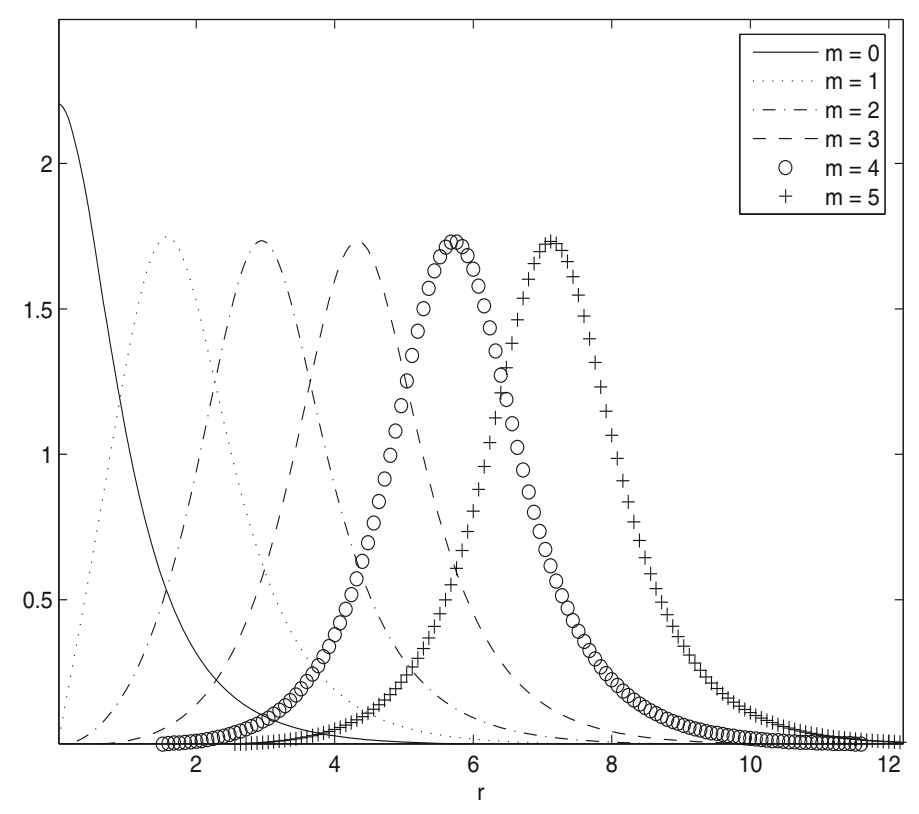

Figure 7. Plots of the zero node vortex, $m=0,1,2,3,4,5(\alpha=1, \sigma=1)$.

We also use a shooting strategy in this new context in order to find stationary states denoted by $u_{k, m}$ (that is with $k$ nodes and corresponding to the winding number $m$ ). These vortex states have been theoretically studied in [14] in the case $k=0$ and in [8] for any $k$. We show the results obtained in the cubic focusing case $\sigma=1, \omega=1, \alpha=1$ : in Figure 7 are shown the profiles of the nodeless solutions (but that vanish at $r=0$; the $k$ th excited state is defined here as the solution that vanishes $k$ times outside the origin), computed with $m=0, m=1, m=2, m=3, m=4$ and $m=5$ whereas in Figure 8 is shown the spatial profile of the vortex computed with $(k, m)=(0,1)$. It can be clearly pointed out in Figure 7 that solitons obtained for large $m$ look like spatially translated soliton $u_{0,1}$. In particular for $m \geq 1$, the maximal amplitude does not seem to depend on $m$ and the distance between two consecutive maxima is approximately 1.4. We also plot in Figure 9 excited vortices with $m=1$ obtained for various $k(k=0,1,2,3)$ and in Figure 10 the spatial profile obtained with $(k, m)=(1,3)$.

\subsection{Case of negative power index}

We also numerically investigate the particular case $-1 / 2<\sigma<0$ for which the refraction index $|u|^{2 \sigma}$ is no more defined but the nonlinearity still makes sense. We use the numerical algorithm described above to compute the corresponding solitons in this context. We have taken the parameters $d=2, \sigma=-1 / 4$ and $\alpha=\omega=-1$ (that is the defocusing case that appears to be the only way to grant existence of such solutions). In Figure 11 are plotted the four solutions obtained with $N=1000$ on a numerical bounded domain depending on the node number. Even if the profile does not much differ from the ones already displayed, there is a striking difference that can be noticed when viewing Figure 12 the log profile of these states with respect to $r$. In this case, the log profiles do not decrease almost linearly as in Figure 6 after the last node: this suggests that the approximate solutions tend to zero much faster than with the usual exponential rate. Since tests performed with larger values of $N$ on the same interval have shown log profiles that do not stabilize around these transition zones, it is thus conjectured that these states are the compactly supported solutions that have been obtained in [1] for sublinear elliptic problems. Since $0<2 \sigma+1<1$, the nonlinear term $\alpha|u|^{2 \sigma} u$ is not negligible with respect to the other ones and the large distance asymptotics $u(r) \equiv C \mathrm{e}^{-r} / \sqrt{r}$ found for $\sigma>0$ is no more valid. 


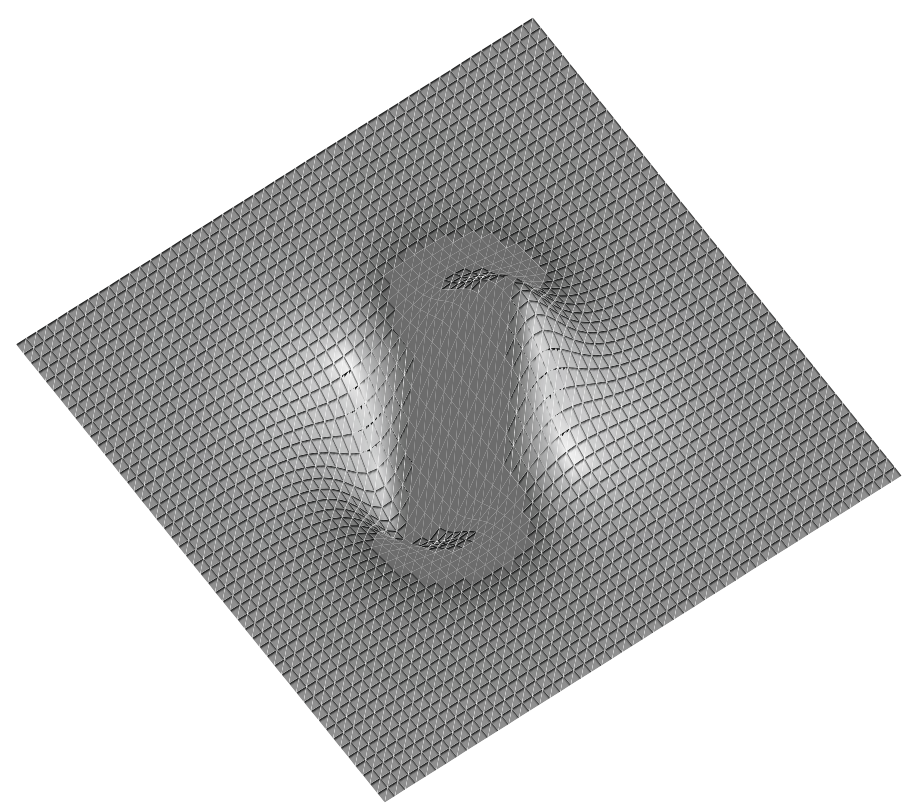

Figure 8. Cartesian $(x, y)$-plot of Re $\left(\mathrm{e}^{\mathrm{i} m \theta} u_{k, m}(r)\right),(k, m)=(0,1)$.

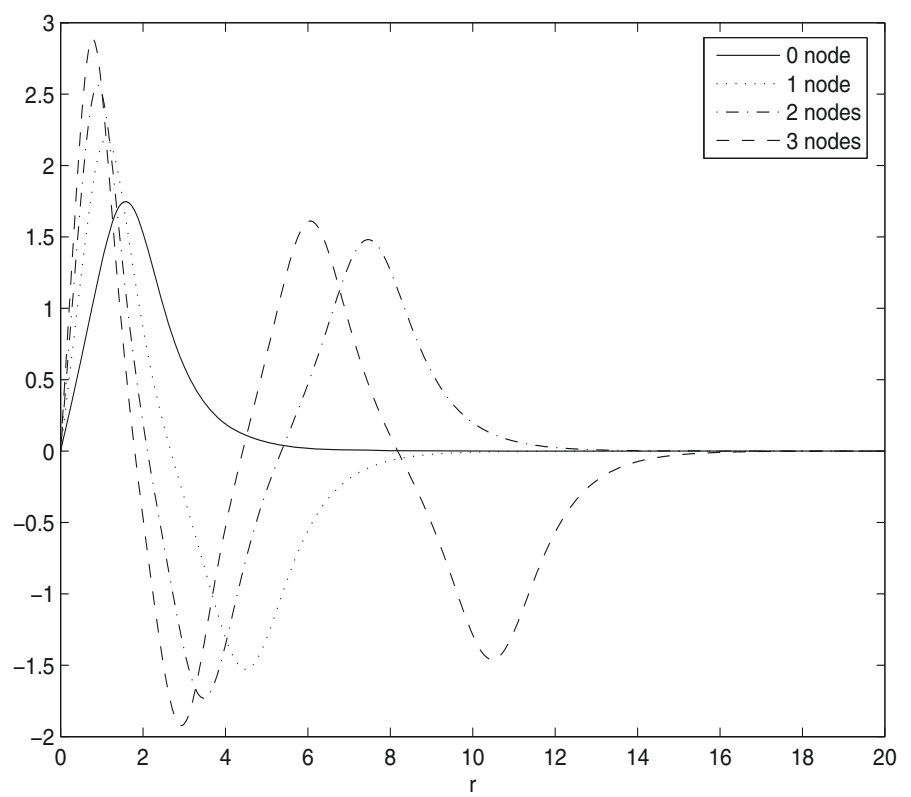

Figure 9. Plots of the first four vortex solutions, $m=1(\alpha=1, \sigma=1)$.

\subsection{Asymptotics}

We now intend to investigate the asymptotic behaviour the excited states as the number of nodes increases. Indeed, it has been found in [9] that the $L^{2}$ and $H^{1}$ norms of $u_{k}$ asymptotically behave as $k^{d / 2}$ under suitable assumptions made on the nonlinearity. In [15], the $k$ th energy level for the Schrödinger-Newton equation, 


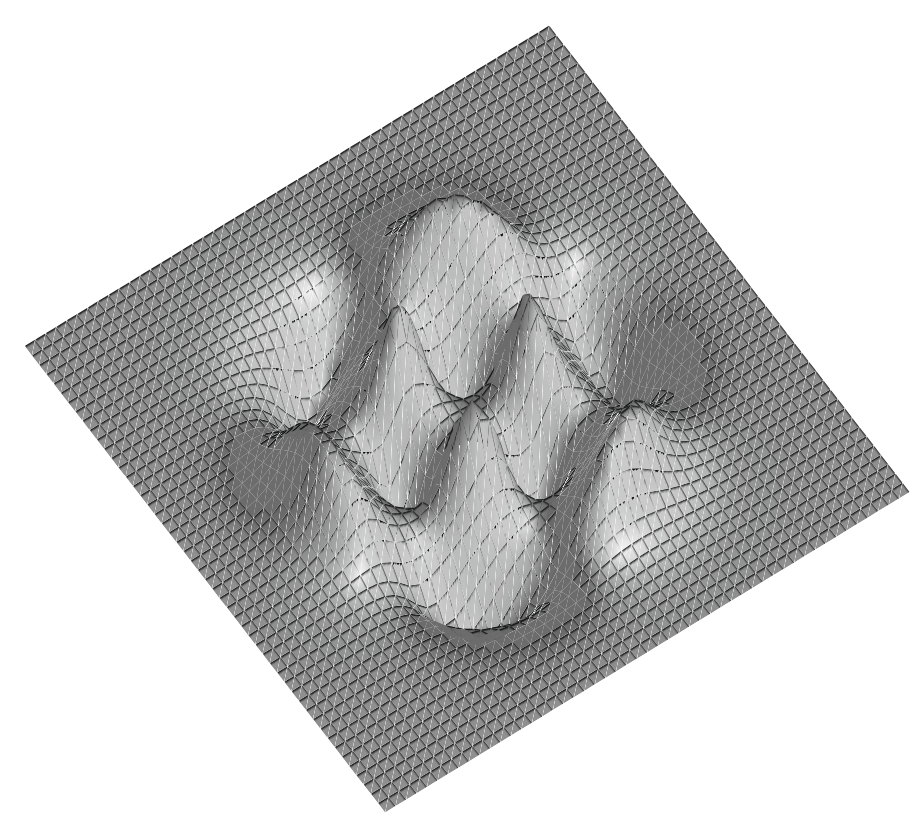

Figure 10. Cartesian $(x, y)$-plot of $\operatorname{Re}\left(\mathrm{e}^{\mathrm{i} m \theta} u_{k, m}(r)\right),(k, m)=(1,3)$.

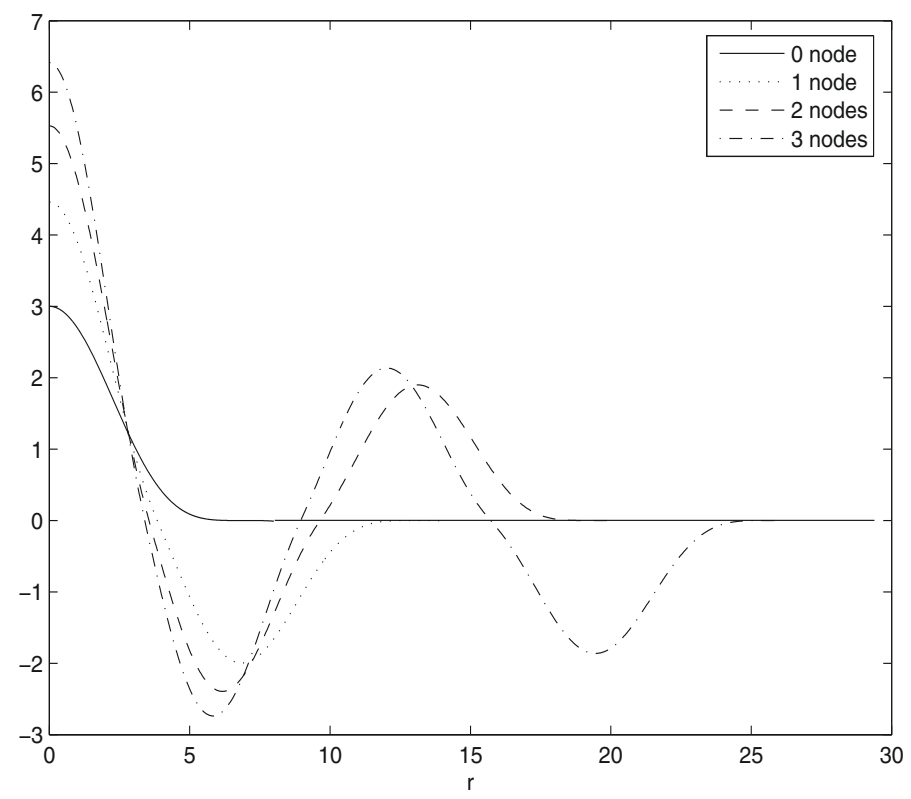

FIGURE 11. Plots of the solitons computed with $k=0, k=1, k=2$ and $k=3(d=2$, $\alpha=\omega=-1, \sigma=-1 / 4)$.

defined as the frequency $\omega_{k}$ associated to the $L^{2}$ normalized solution of the nonlinear eigenvalue problem has been numerically found to evolve as $k^{-2}$. Our goal is to study the influence of $d, \sigma$ and $m$ (for $d=2$ ) on the stationary excited states with large numbers of nodes. 


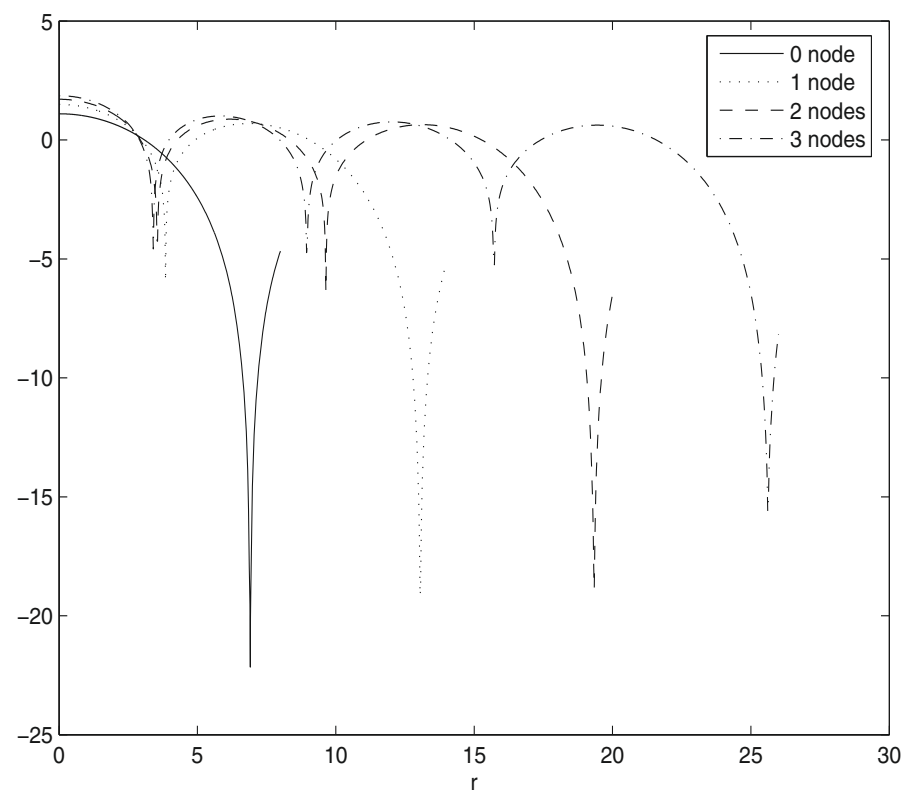

Figure 12. Plots of the logarithm of the soliton profiles $(d=2, \alpha=\omega=-1, \sigma=-1 / 4)$.

\subsubsection{Bright solitons}

First, we have observed that the value $u_{k}(0)$ and $\left\|u_{k}\right\|_{L^{2}}$ seem to increase with $k$ (see Figs. 13 and 14 where these two quantities have been plotted for $0 \leq k \leq 25$ for various $\sigma$ in the two-dimensional case $d=2$ ). In these computations, system (2.1) has been solved on a spatial domain that increases with the number of nodes (we have observed in our all tests that a suitable choice for $R$ in the computation of $u_{k}$ previously described is $R_{k}=R_{0}+k \delta$, where $\delta$ is a constant depending on $\sigma$ ). These two plots show that these two quantities increase for any value of $\sigma$ and the plot of $\left\|u_{k}\right\|_{L^{2}}$ seems to depend linearly on parameter $k$ for any value of $\sigma$.

We now intend to study accurately the dependence of these norms regarded as functions of $k$. Seeking a asymptotic law $f_{k} \sim k^{\gamma}(k \rightarrow \infty)$ for some sequence $f_{k}$ that can be computed for each $k$, it is possible to plot $\log f_{k}$ as a function of $\log k$ that should behave linearly with the slope $\gamma$. This means that the finite difference approximation of the discrete derivative $\delta_{k}:=\log \left(f_{k+1} / f_{k}\right) / \log ((k+1) / k)$ of $\log f_{k}$ with respect to $\log k$ should tend to $\gamma$ for large $k$. Considering first the $L^{\infty}$ norm, the computed derivatives (plotted in Fig. 15) converge to the limit 0.5 for any value of nonlinear exponent $\sigma$. This suggests that the asymptotic behaviour $\left\|u_{k}\right\|_{L^{\infty}} \sim \sqrt{k}$ holds. Note that the same computations made for the $\left\|u_{k}\right\|_{L^{2}}$ and $\left\|\nabla u_{k}\right\|_{L^{2}}$ numerically confirm the asymptotics given by Theorem 1 in [9]: we have found $\left\|u_{k}\right\|_{L^{2}} \sim k,\left\|\nabla u_{k}\right\|_{L^{2}} \sim k$ and $S\left(u_{k}\right) \sim k^{2}$ as $k \rightarrow \infty$.

The same computations have been made for higher values of $d$. Surprisingly, they showed that for $d \geq 3$, the asymptotic growth now depends on $\sigma$ (see Fig. 16 for $d=3$ ), whereas the $L^{2}$ norm of the solution still obeys the asymptotics $\left\|u_{k}\right\|_{L^{2}} \sim k^{d / 2}$ given in [9]. Our results have shown that $S\left(u_{k}\right) \sim k^{d}$ that is again consistent with [9]. Let us mention that for large values of $\sigma$ (that means $\sigma \rightarrow \sigma^{*}$ ), it becomes quite difficult to find accurate values for excited solitons since the values at the origin become very large (as an example, for $k=25$ and $d=4$, we have found $u_{0}^{k} \simeq 1.5040 \times 10^{7}$ ). All these experiments suggest us that for $d \geq 2$, the asymptotics of the $L^{\infty}$ norm of the solutions is governed by some power law

$$
\left\|u_{k}\right\|_{L^{\infty}} \sim k^{\gamma(d, \sigma)}
$$

where $\gamma(2, \sigma)=1 / 2, \forall \sigma>0$ and where $\gamma(d,$.$) is an increasing function of \sigma$ if $d \geq 3$. (Moreover, Fig. 16 suggests that $\gamma$ is nonlinear with respect to $\sigma$.) In order to find the profile of $\gamma$, we compute the limit value 


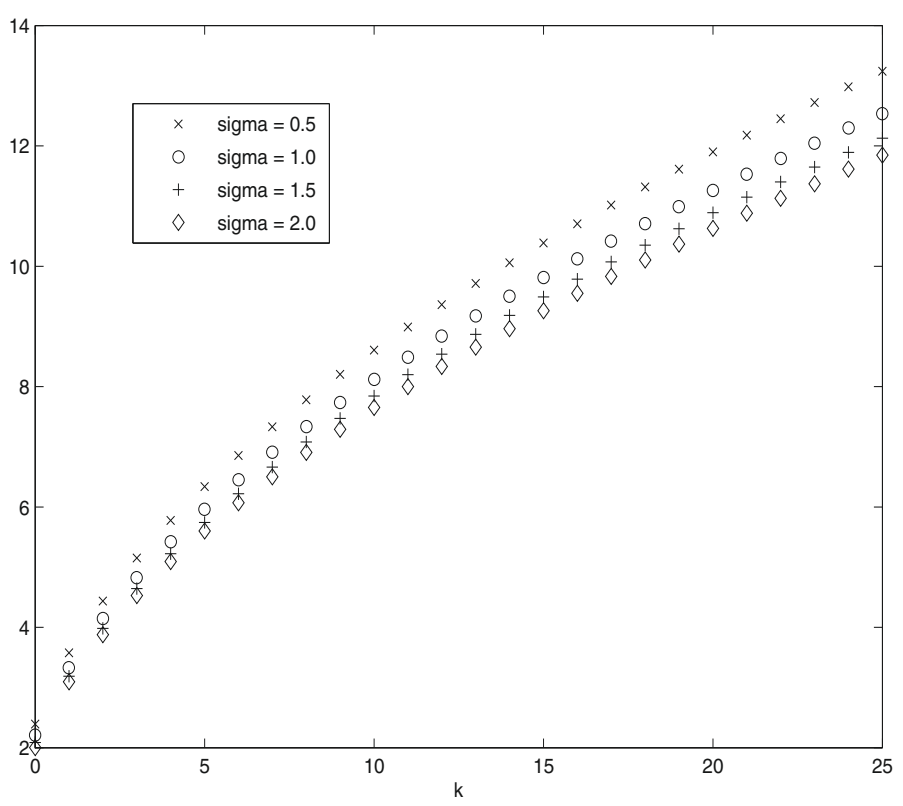

Figure 13. $u_{0}^{k}$ for $0 \leq k \leq 25, \sigma=0.5,1.0,1.5,2.0(d=2)$.

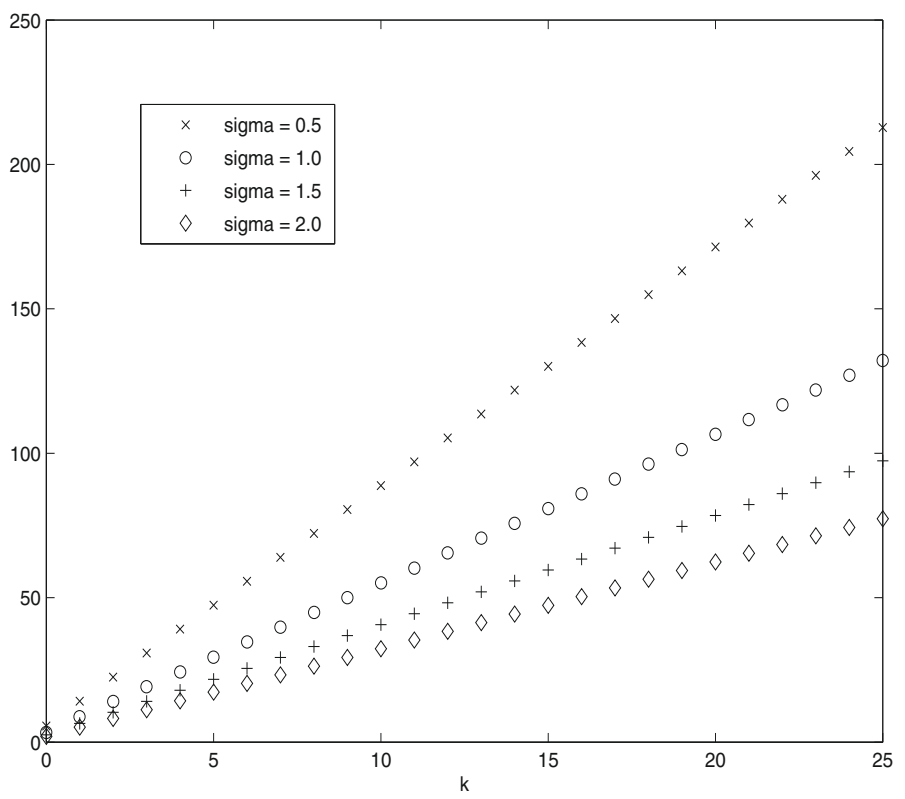

FiguRE 14. $\left\|u_{k}\right\|_{L^{2}}$ for $0 \leq k \leq 25, \sigma=0.5,1.0,1.5,2.0(d=2)$.

of the derivative when $\sigma$ continuously varies from 0 to $\sigma^{*}$ and it is found that $\gamma$ diverges when $\sigma$ tends to $\sigma^{*}$. We then study $\log \gamma(d, \sigma)$ as a function of $\log \left(\sigma^{*}-\sigma\right)$ and observe a slope very close to -1 for every $d$; we thus set

$$
\gamma(d, \sigma)=\frac{C(d)}{\sigma^{*}-\sigma}, \sigma \in\left[0, \sigma^{*}[.\right.
$$




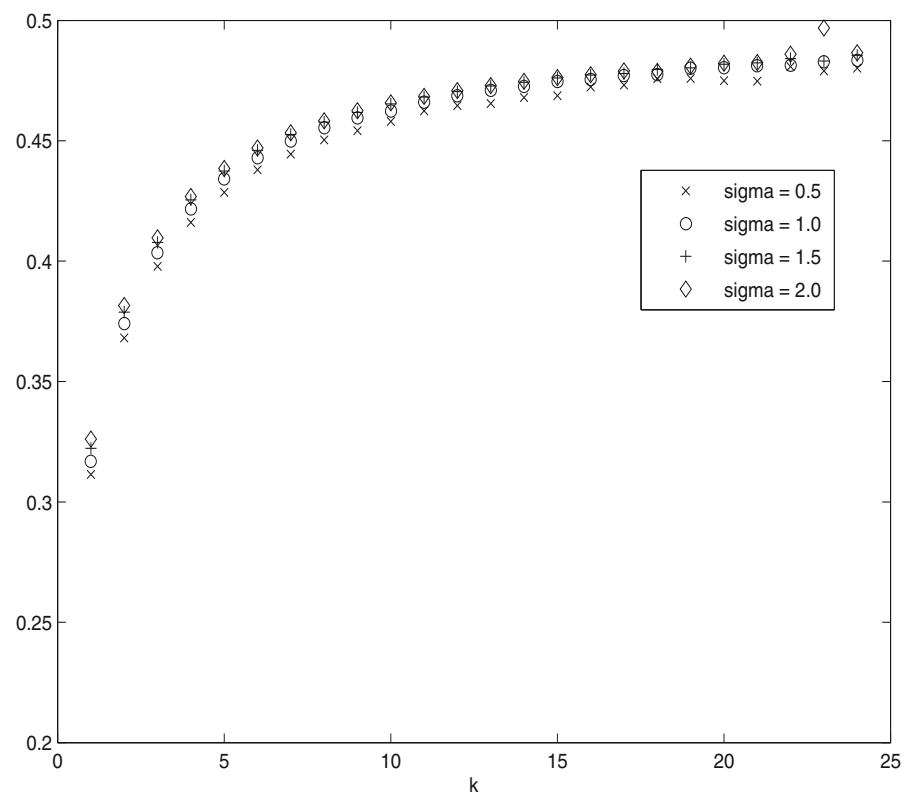

FiguRE 15. Derivative of $\log u_{0}^{k}$ as a function of $\log k$ for $0 \leq k \leq 25, \sigma=0.5,1.0,1.5,2.0(d=2)$.

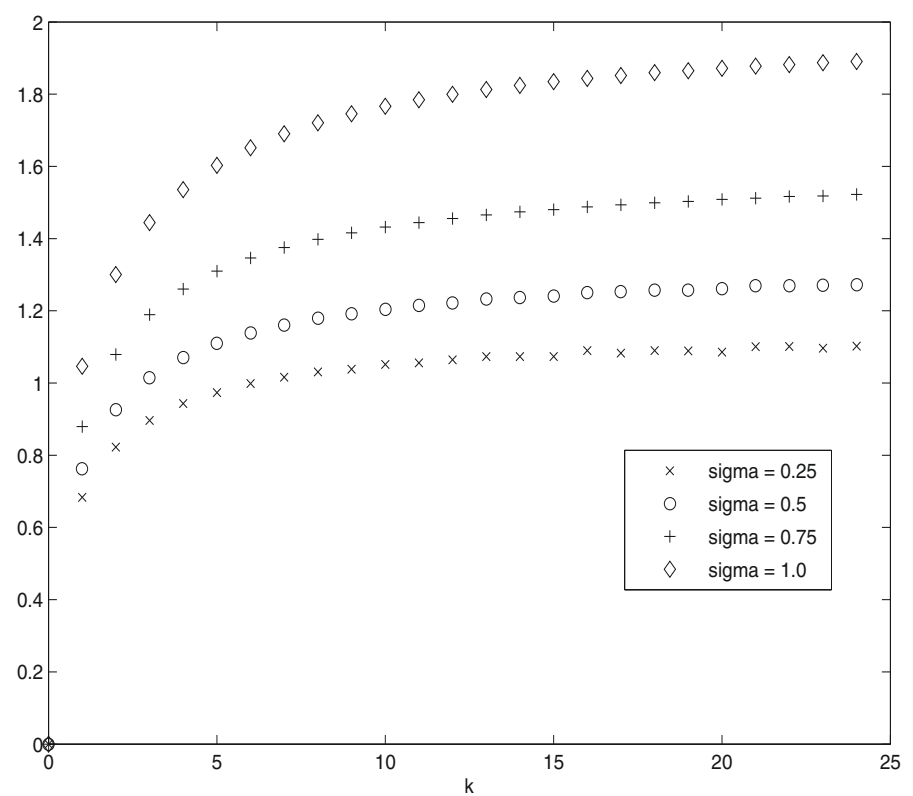

Figure 16. Derivative of $\log u_{0}^{k}$ as a function of $\log k$ for $0 \leq k \leq 25, \sigma=0.25,0.5,0.75,1.0$ $(d=3)$.

The constant $C(d)$ is then numerically found by comparing the numerical profiles of $\gamma(d, \sigma)$ with the desired expression. It has been found in all our tests that $C(d)$ closely looks like the constant $(d-1) /(d-2)=1+\sigma^{*} / 2$. 


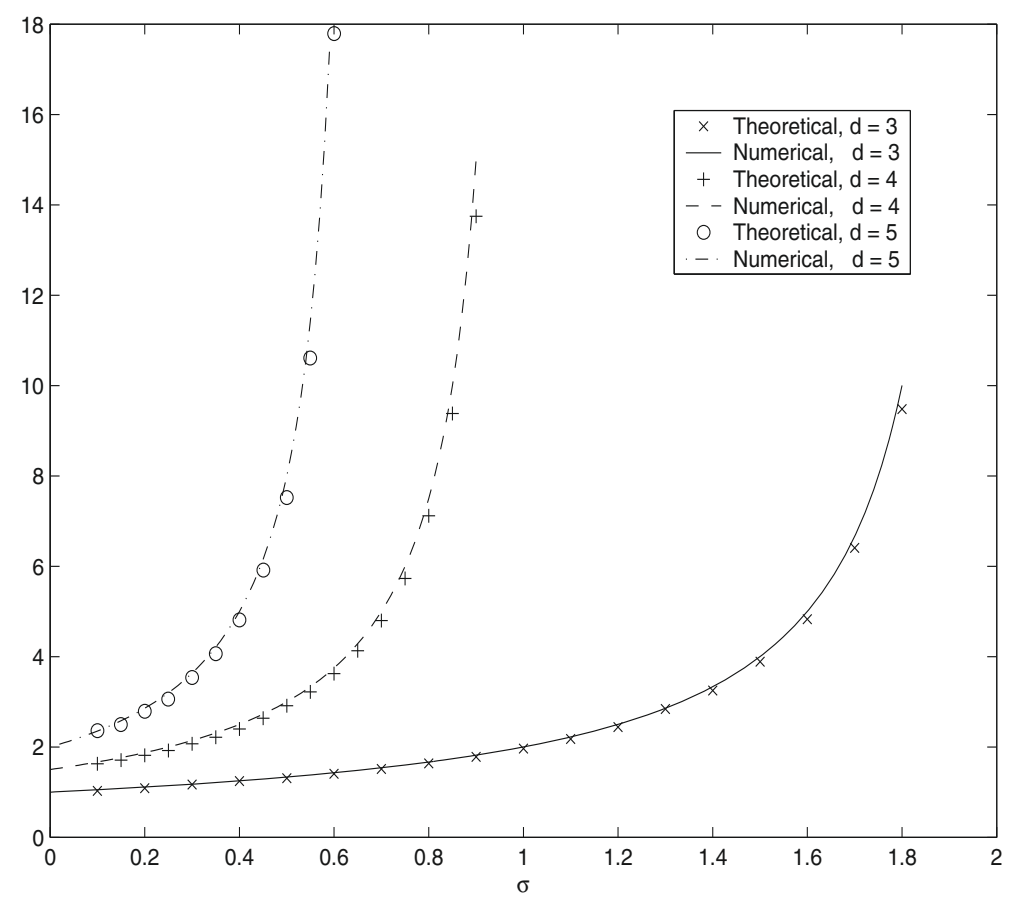

Figure 17. Comparison between numerical and possible theoretical values of $\gamma(d, \sigma), \sigma \in$ $\left[0, \sigma^{*}[, d=3,4,5\right.$.

We thus conjecture that the $L^{\infty}$ norm of the $k$ th excited state obeys the asymptotic power law

$$
\left\|u_{k}\right\|_{L^{\infty}} \sim k^{\frac{1+\sigma^{*} / 2}{\sigma^{*}-\sigma}}=k^{\frac{d-1}{2+\sigma(2-d)}}, k \rightarrow \infty
$$

This expression still makes sense for $d=2$ : we then have $\sigma^{*}=\infty$ and this implies $\gamma(2, \sigma)=1 / 2$ that had been observed below. Figure 17 displays a remarkably good agreement between the computed $\gamma$ for different $\sigma$ and $d$ and the expression given in (2.3), recalling that each value of $\sigma$ is obtained as a numerically calculated limit when $k$ is large enough.

We finally made the same tests in the case of negative index in order to look again for asymptotic rates in various norms. The situation now differs from what was found above: the limit value of the derivative of $\log \left\|u_{k}\right\|_{L^{\infty}}$ with respect to $\log k$ does not depend on $\sigma$. The profiles obtained indicate that we have the asymptotics

$$
\left\|u_{k}\right\|_{L^{\infty}} \sim k^{\frac{d-1}{2}},\left\|u_{k}\right\|_{L^{2}} \sim k^{d / 2},\left\|\nabla u_{k}\right\|_{L^{2}} \sim k^{d / 2} \text { and } S\left(u_{k}\right) \sim k^{d}
$$

\subsubsection{Vortex solitons}

We now set $d=2$ and look for asymptotics laws in $L^{2}, H^{1}$ norms of vortex solutions as $k$ is large. It is now observed (see Figs. 18 and 19) that for each $m \geq 1$, the value $U_{0}^{k, m}$ of $U_{k, m}(r):=u_{k, m}(r) / r^{m}$ at $r=0$ depends on both $\sigma$ and $m$. It has also been obtained that the $L^{2}$ norm of $u_{k, m}$ uniformly scales as $k$. We notice that in this case, the rate that is numerically found for the $L^{\infty}$ norm seems to fit the explicit one given by

$$
U_{0}^{k, m} \sim k^{\frac{1+m \sigma}{2}}, k \rightarrow \infty
$$




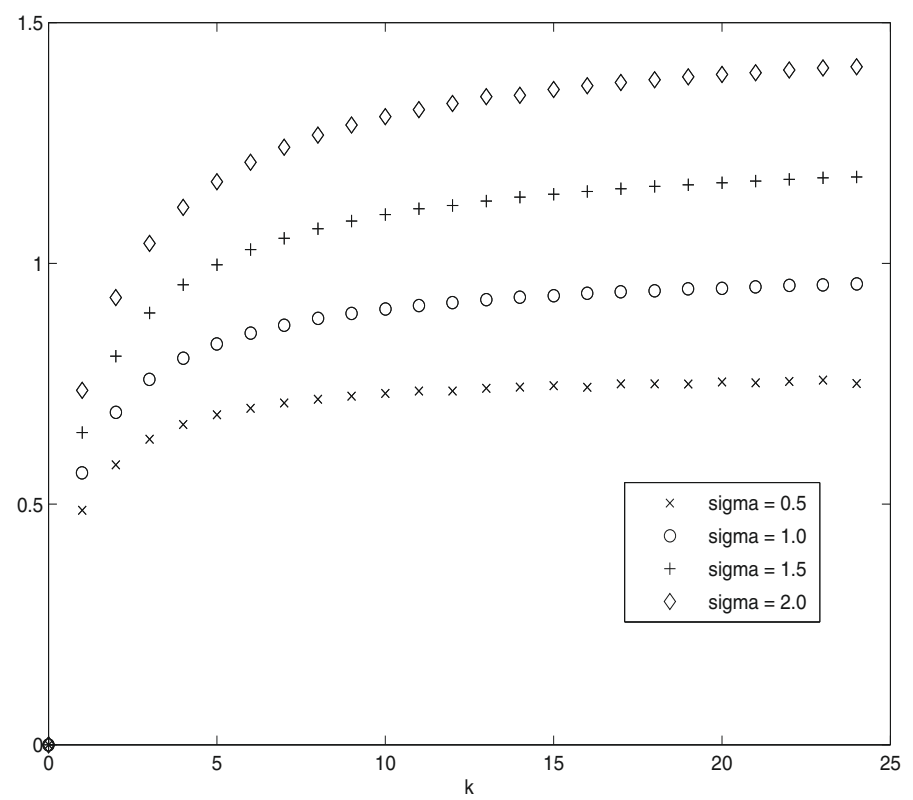

Figure 18. Derivative of $\log U_{0}^{k, m}$ as a function of $\log k$ for $0 \leq k \leq 25, \sigma=0.25,0.5,0.75,1.0$ $(m=1)$.

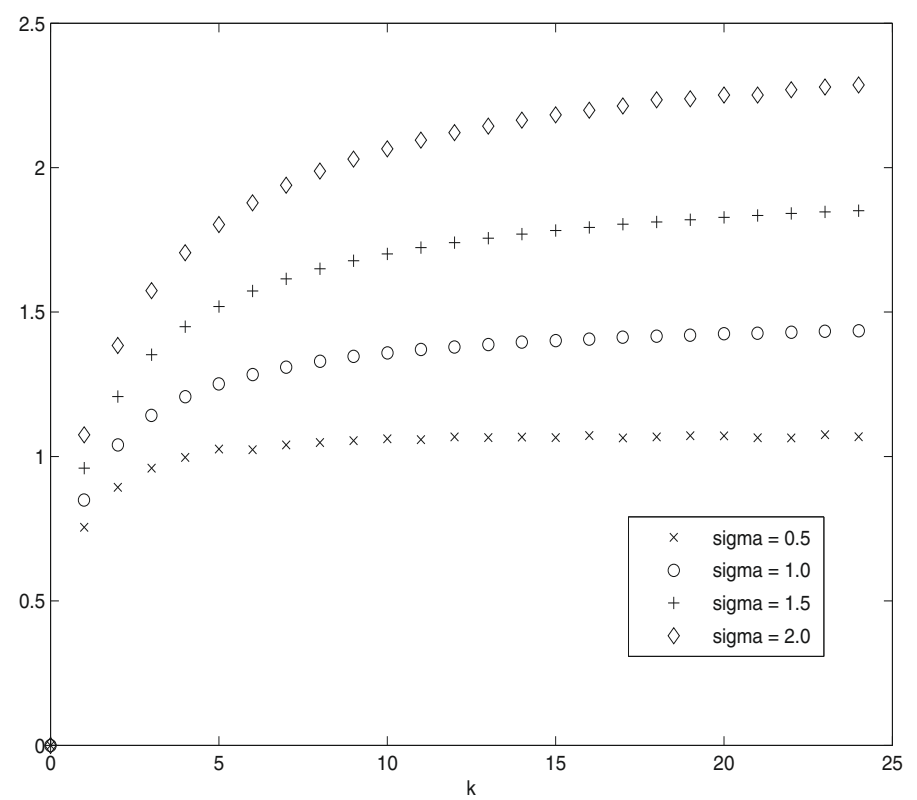

FiguRE 19. Derivative of $\log U_{0}^{k, m}$ as a function of $\log k$ for $0 \leq k \leq 25, \sigma=0.25,0.5,0.75$ $(m=2)$.

\subsubsection{Large nonlinear exponent}

As recalled in the beginning of Section 2.1, solitons can be computed when $\alpha>0$ and $\omega>0$ only for $\sigma<\sigma^{*}$ and we now focus on the asymptotics $\sigma \rightarrow \sigma^{*}$ : we compute stationary states for large values of $\sigma$ in order 


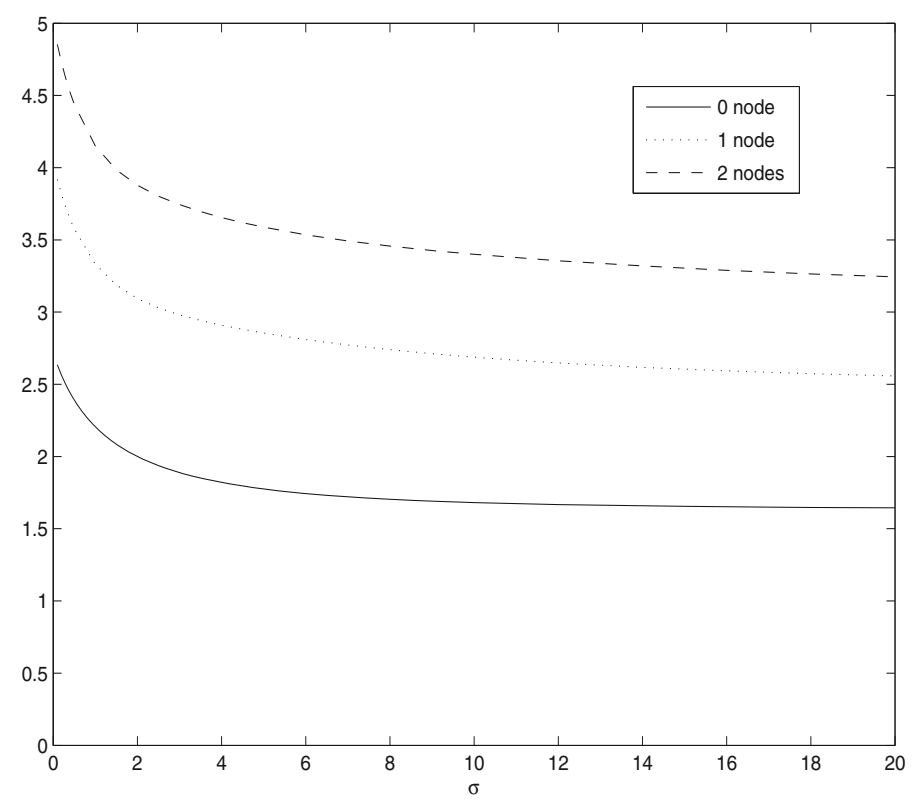

Figure 20. Values of $\left\|u_{0}\right\|_{L^{\infty}},\left\|u_{1}\right\|_{L^{\infty}}$ and $\left\|u_{2}\right\|_{L^{\infty}}$ versus $\sigma(d=2)$.

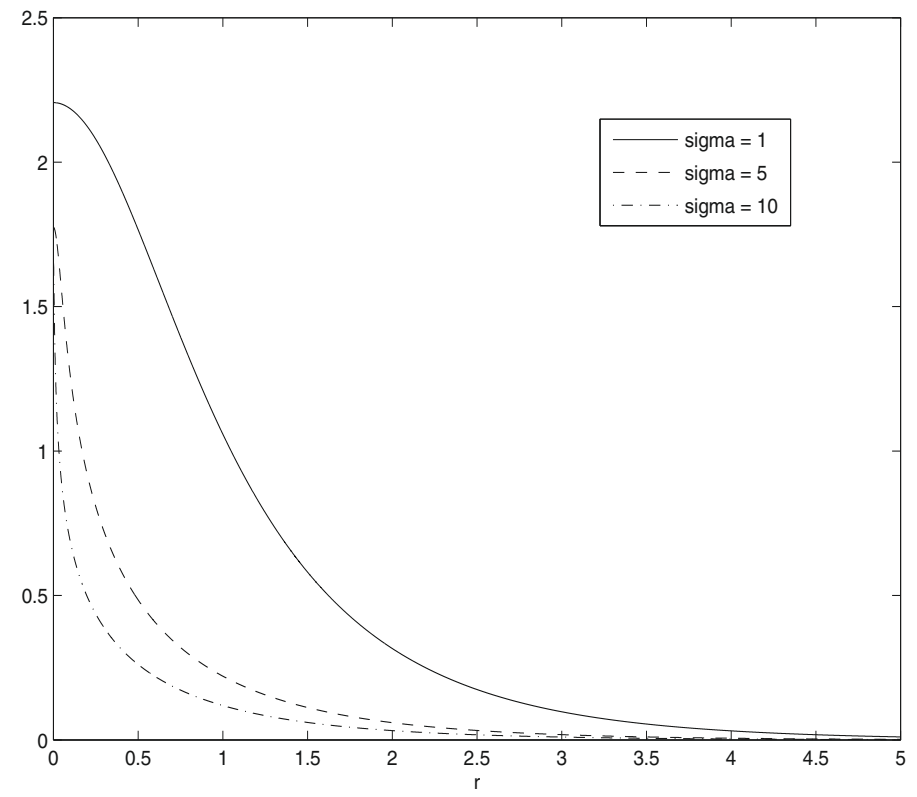

Figure 21. Plot of $u_{0}, \sigma=1, \sigma=5, \sigma=10(d=2)$.

to derive a scaling law for $\left\|u_{k}\right\|_{L^{\infty}},\left\|u_{k}\right\|_{L^{2}},\left\|\nabla u_{k}\right\|_{L^{2}}$ and $S\left(u_{k}\right)$ when $k$ is prescribed. Our experiments first show that for $d=2$, the value of the soliton at the origin seems to converge as $\sigma \rightarrow \infty$ to some limit depending on the number of nodes (see Fig. 20 where this value is plotted with respect to $\sigma$ for $k=0, k=1$ and $k=2$ ). Furthermore, a spatial plot of the fundamental solution $u_{0}$ for various $\sigma$ indicates a confinement of the stationary state around the origin when $\sigma \rightarrow \infty$, as viewed in Figure 21. 


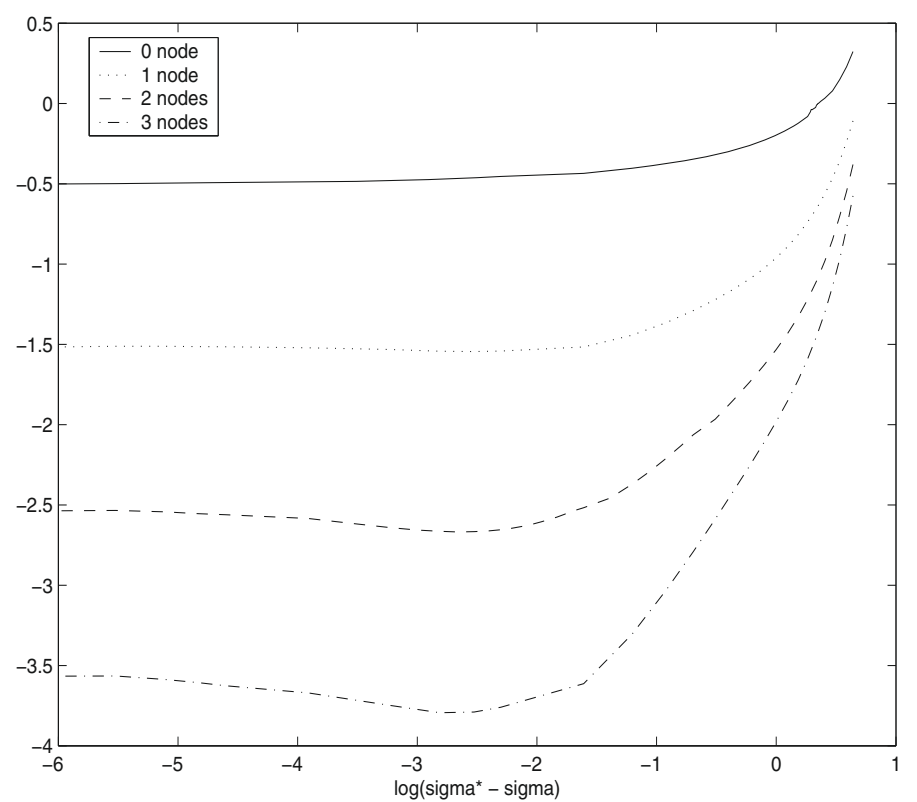

Figure 22. Derivative of $\log \left\|u_{k}\right\|_{L^{\infty}}$ as a function of $\log \left(\sigma^{*}-\sigma\right)(d=3), k=0, k=1, k=2$ and $k=3$.

In this case, we have used a differential solver on a nonuniform grid that is geometrically refined around $r=0$ : we have set $r_{j}=\lambda^{j} r_{0}$, with $\lambda>1$ constant. All the experiments have been made with $\lambda=1.01$ that is enough to capture the singularity. Our computations showed that there does not exist a uniform scaling factor $\lambda_{k}(\sigma)$ for which $u_{k}\left(\lambda_{k}(\sigma) r\right)$ could tend to a universal profile as in the one-dimensional case for the ground state. Higher-order schemes have been used to check the validity of the results obtained with RK4 method in the resolution of (2.1); it has been observed that the computed solutions were not significantly changed, leading us to the conclusion that taking a standard fourth-order differential solver on a refined grid around the origin was sufficient to approximate accurately localized states. We now consider the case $d \geq 3$ for which $\sigma^{*}<\infty$. We perform the same experiments as before and observe that $u_{0}^{k}$ tends to infinity as $\sigma \rightarrow \sigma^{*}$. We intend to analyze the rate of blow-up of this quantity in terms of $\sigma^{*}-\sigma$ : as before, we view the derivative of log $u_{0}^{k}$ as depending on $\log \left(\sigma^{*}-\sigma\right)$ (see Fig. 22 for $d=3$ ) for the first four excited states $u_{0}, u_{1}, u_{2}$ and $u_{3}$.

It can be observed that in any dimension, the rate of convergence depends on the number of nodes: it seems that large $k$ give a stronger tendency to diverge. We give an illustration of this property in Figure 22 where the approximate rate of blow-up is given as $\sigma$ tends to $\sigma^{*}$ for $d=3$. Longer tests made with different $d$ and $k$ have shown that this rate seems to behave as $-\frac{1}{2}-k$. We thus conjecture that

$$
\left\|u_{k}\right\|_{L^{\infty}} \sim\left(\sigma^{*}-\sigma\right)^{-\frac{1}{2}-k}, \sigma \rightarrow \sigma^{*}
$$

Our experiments have shown that the $L^{2}$ norm of $u_{k}$ tends to zero with the uniform rate $\left(\sigma^{*}-\sigma\right)^{\frac{1}{2}}$ that do not not depend neither on $k$, neither on the spatial dimension. Furthermore, the profile of the limit value of $S\left(u_{k}\right)$ as $\sigma \rightarrow \sigma^{*}$ for different $d$ with respect to $k$ behaves as a linear function of $k$. We may have in the general case

$$
S\left(u_{k}\right) \sim c_{k} \sim k, \sigma \rightarrow \sigma^{*}
$$

We have plotted in Figure 23 the values found for the derivative of the limit of $\left\|\nabla u_{k}\right\|_{L^{2}}$ with respect to log $k$ for $0 \leq k \leq 15$ and $3 \leq d \leq 5$. In all dimensions, this derivative converges to $1 / 2$. This result suggests that 


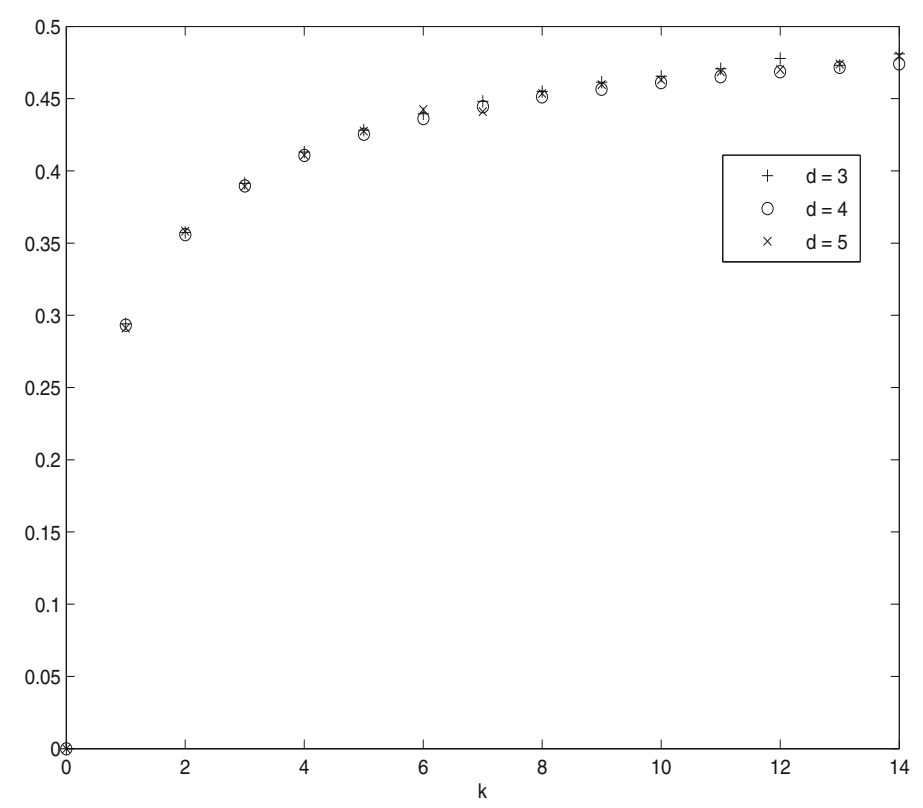

Figure 23. Limit values of the derivative of $\log \left\|\nabla u_{k}\right\|_{L^{2}}$ with respect to $\log \left(\sigma^{*}-\sigma\right)$ versus $k$, $d=3,4,5$.

for any dimension, we have

$$
\left\|\nabla u_{k}\right\|_{L^{2}} \sim d_{k} \sim \sqrt{k}, \sigma \rightarrow \sigma^{*} .
$$

We have also noticed that in the case $d=3$, the profile of $\left\|u_{k}\right\|_{L^{\infty}}$ is not monotonous with respect to $\sigma$ : we have found that this quantity decreases from zero to some $\tilde{\sigma}$ and increases from $\tilde{\sigma}$ to $\sigma^{*}$. The numerical value of $\tilde{\sigma}$ is 0.585 , which is not far from the critical exponent $2 / d$ in that case. For higher dimensions, the profile of the $L^{\infty}$ norm is always increasing with respect to $\sigma$.

\subsubsection{Sum up of the results}

We finally recall all the asymptotics obtained for the stationary states in the previous paragraphs.

- For $\sigma<\sigma^{*}=2 /(d-2)$ in the case $d \geq 2, \omega=1$ and $\alpha=1$, we have for $k \rightarrow \infty$

$$
\left\|u_{k}\right\|_{L^{\infty}} \sim k^{\frac{1+\sigma^{*} / 2}{\sigma^{*}-\sigma}},\left\|u_{k}\right\|_{L^{2}} \sim k^{d / 2},\left\|\nabla u_{k}\right\|_{L^{2}} \sim k^{d / 2} \text { and } S\left(u_{k}\right) \sim k^{d}
$$

- For $\sigma>0$ in the case $d=2, \omega=1$ and $\alpha=1$, we have for $k \rightarrow \infty$

$$
\left.\frac{u_{k, m}}{r^{m}}\right|_{r=0} \sim k^{\frac{1+m \sigma}{2}},\left\|u_{k, m}\right\|_{L^{2}} \sim k,\left\|\nabla u_{k, m}\right\|_{L^{2}} \sim k \text { and } S\left(u_{k}\right) \sim k^{2} .
$$

- For $-1 / 2<\sigma<0$ in the case $d \geq 2, \omega=-1$ and $\alpha=-1$, we have for $k \rightarrow \infty$

$$
\left\|u_{k}\right\|_{L^{\infty}} \sim k^{\frac{d-1}{2}},\left\|u_{k}\right\|_{L^{2}} \sim k^{d / 2},\left\|\nabla u_{k}\right\|_{L^{2}} \sim k^{d / 2} \text { and } S\left(u_{k}\right) \sim k^{d} .
$$

- For $d \geq 2, \omega=1$ and $\alpha=1$, we have at prescribed $k$ when $\sigma \rightarrow \sigma^{*}=2 /(d-2)$

$$
\left\|u_{k}\right\|_{L^{\infty}} \sim\left(\sigma^{*}-\sigma\right)^{-\frac{1}{2}-k},\left\|u_{k}\right\|_{L^{2}} \sim\left(\sigma^{*}-\sigma\right)^{\frac{1}{2}},\left\|u_{k}\right\|_{L^{2}} \sim \sqrt{k} \text { and } S\left(u_{k}\right) \sim k .
$$




\section{Solitons For TWO-WAVE QUADRATIC MEDia}

We are now concerned with the computation of solitons for a system occurring in nonlinear optics that governs the propagation of two electromagnetic waves in a quadratic media. The system writes

$$
\left\{\begin{array}{l}
i \frac{\partial u}{\partial z}+\Delta u-u+\bar{u} v=0 \\
i \sigma \frac{\partial v}{\partial z}+\Delta v-\rho v+\frac{1}{2} u^{2}=0
\end{array}\right.
$$

where $\sigma$ and $\rho$ stand for nonnegative parameters. Seeking stationary states of the form $u(z, x)=\mathrm{e}^{\mathrm{i} \omega z} u(x)$ and $v(z, x)=\mathrm{e}^{2 \mathrm{i} \omega z} v(x)$, system (3.1) is transformed into the nonlinear elliptic system in $\mathbb{R}^{d}$

$$
\left\{\begin{array}{l}
-(1+\omega) u+\Delta u+\bar{u} v=0 \\
-(\rho+2 \sigma \omega) v+\Delta v+\frac{1}{2} u^{2}=0 .
\end{array}\right.
$$

In all that follows, we will assume for the sake of convenience that $\omega=0$ without loss of generality (taking $\omega \neq 0$ only changes the values of the zero-order parameters). Looking for radial solutions, this system will consist in two differential second-order equations where the unknowns $u$ and $v$ have to be computed for $r \in[0, \infty[$. In this case, the previous scalar shooting method based on a single dichotomy algorithm cannot be used directly because two independent parameters $u(0)$ and $v(0)$ have to be adjusted in order to enforce a nice decay property at infinity. We first focus on the case $d=1$.

\subsection{The one-dimensional case}

If we assume that $u$ and $v$ stand for even functions of $x$, smoothness assumption requires that the two derivatives have to vanish at $x=0$. System (3.2) thus reduces to

$$
\left\{\begin{array}{l}
u^{\prime \prime}(x)-u(x)+u(x) v(x)=0 \quad x>0 \\
v^{\prime \prime}(x)-\rho v(x)+\frac{1}{2} u^{2}(x)=0 \\
u(0)=\alpha, \quad v(0)=\beta, \quad u^{\prime}(0)=0, \quad v^{\prime}(0)=0 .
\end{array}\right.
$$

Proposition 3.1. Let $(\alpha, \beta)$ the initial values of a spatially localized solution $(u, v)$ of (3.3) at $x=0$. Then one has the identity

$$
\alpha^{2}+\rho \beta^{2}-\alpha^{2} \beta=0 .
$$

Proof. We multiply the first equation by $u^{\prime}$ and the second one by $v^{\prime}$. Summing these two relations, one obtains

$$
-\frac{1}{2}\left(u^{2}(x)\right)^{\prime}-\frac{\rho}{2}\left(v^{2}(x)\right)^{\prime}+\frac{1}{2}\left(\left(u^{\prime}\right)^{2}(x)\right)^{\prime}+\frac{1}{2}\left(\left(v^{\prime}\right)^{2}(x)\right)^{\prime}+u(x) u^{\prime}(x) v(x)+\frac{1}{2} u^{2}(x) v^{\prime}(x)=0 .
$$

We now integrate between 0 and $x$ and plug the initial conditions to get (claiming that $u u^{\prime} v+u^{2} v^{\prime} / 2=\left(u^{2} v\right)^{\prime} / 2$ )

$$
\frac{1}{2} u^{2}(x)+\frac{\rho}{2} v^{2}(x)-\frac{1}{2} u^{2}(x) v(x)=\frac{1}{2} \alpha^{2}+\frac{1}{2} \rho \beta^{2}-\frac{1}{2} \alpha^{2} \beta .
$$

Now, since we look for localized solutions for (3.3), we assume that $u, v$ and all their derivatives vanish at infinity. Letting $x$ tend to infinity, the result follows. 


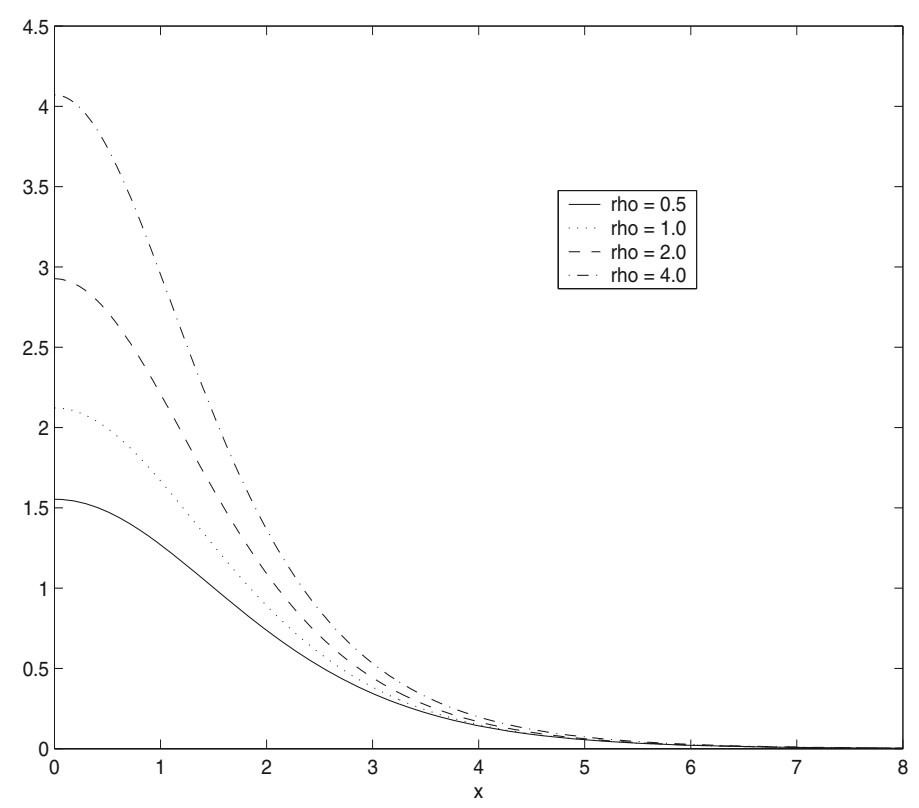

Figure 24. Plot of $u_{0}, \rho=0.5, \rho=1, \rho=2$ and $\rho=4$.

As a consequence, it is possible to express one of the two parameters in terms of the other one, which enables us to define a shooting strategy as for the case of a single equation. We have equivalently

$$
\alpha= \pm \beta \sqrt{\frac{\rho}{\beta-1}} \text { or } \beta=\frac{\alpha^{2} \pm \alpha \sqrt{\alpha^{2}-4 \rho}}{2 \rho} \text {. }
$$

Note that if $(u, v)$ solves $(3.2)$, so does $(-u, v)$ and it is possible to make the further assumption $\alpha>0$. Moreover, in order to have definite square roots, we require that $\beta>1$ and $\alpha>2 \sqrt{\rho}$. If the values at the origin are seen as local minima of $u$ and $v$, then $u^{\prime \prime}(0) \leq 0, v^{\prime \prime}(0) \leq 0$ and (3.3) implies that $\rho \beta \leq \alpha^{2} / 2$, from which we deduce that we finally have $\beta=\left(\alpha^{2}-\alpha \sqrt{\alpha^{2}-4 \rho}\right) / 2 \rho$. Note that the condition $\rho \beta \leq \alpha^{2} / 2$ leads us to $\beta \leq 2$ and we find that $\beta$ is uniformly bounded. We have always chosen to express $\beta$ with use of $\alpha$ since in the second equation, the maximum principle asserts that $v$ remains strictly positive: $\alpha$ will be adjusted in order to obtain a solution which can vanish $k$ times. The $k$ th excited solutions will be noted $\left(u_{k}, v_{k}\right)$, with values at $x=0$ given by $\left(u_{0}^{k}, v_{0}^{k}\right)$.

We plot in Figures 24 and 25 the fundamental solutions $\left(u_{0}, v_{0}\right)$ obtained with the shooting technique for different values of $\rho$ on the domain $[0,10]$ using the Runge-Kutta method with $N=1000$. As opposed to NLS equation (1.1), it can be observed that the values $\left(u_{0}^{k}, v_{0}^{k}\right)$ of the $k$ th excited solution at $x=0$ (that is the solution of (3.3) for which $u$ vanishes $k$ times) seem to converge to a limit value $\left(u^{*}, v^{*}\right)$ that is conjectured to be the Cauchy data of the periodic solution of (3.3): we present in Table 1 the values $u_{0}^{k}$ and $v_{0}^{k}$ obtained for $0 \leq k \leq 5$ in the case $\rho=0.5$.

Consequently, it becomes quite difficult to analyze the rate of convergence of $\left(u_{0}^{k}, v_{0}^{k}\right)$ to $\left(u^{*}, v^{*}\right)$. We also view in Figures 26 and 27 the profiles of the first excited states. We remark that $v_{k}$ can be closely approximated by a translated plot of $v_{k-1}$; this provides a numerical illustration that letting $k$ tend to infinity leads us to a periodic solution.

We have also found that excited states $\left(u_{k}, v_{k}\right)$ only seem to exist for $0<\rho<1$. As an example, we try to compute the first excited solution when $\rho$ tends to 1 . The approximate solution $v$ attains a local extremum at some $x_{0}$ that is observed to tend to infinity when $\rho$ becomes close to 1 (see Figs. 28 and 29, where this 


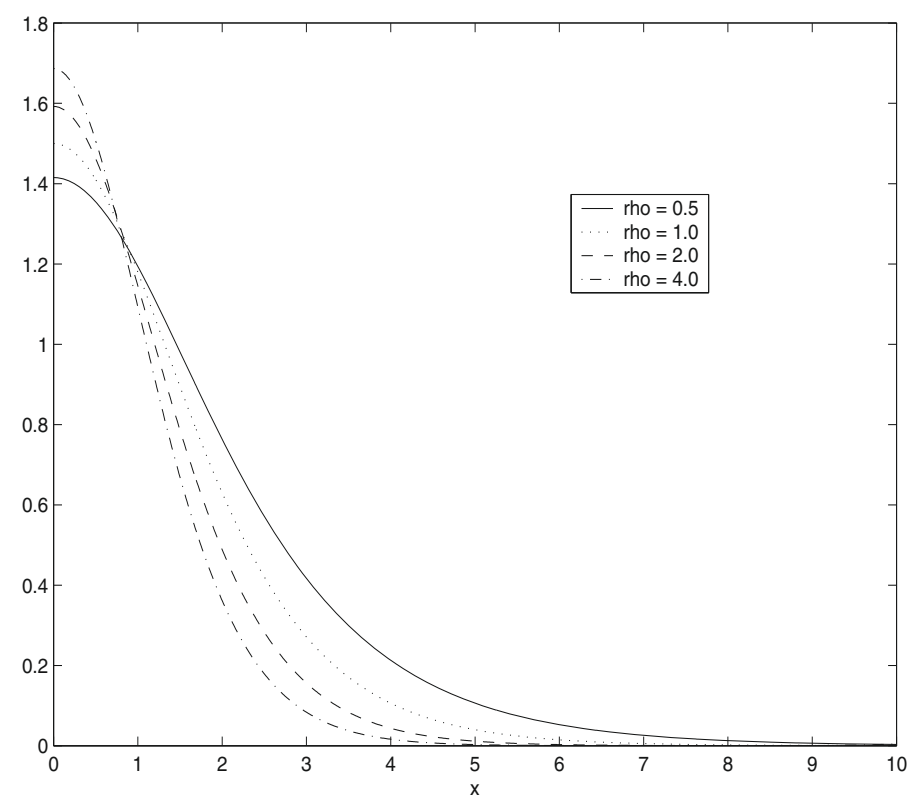

Figure 25. Plot of $v_{0}, \rho=0.5, \rho=1, \rho=2$ and $\rho=4$.

TABle 1. $u_{0}^{k}$ and $v_{0}^{k}$ for $0 \leq k \leq 5$.

\begin{tabular}{c|c|c}
\hline$k$ & $u_{0}^{k}$ & $v_{0}^{k}$ \\
\hline 0 & 1.55309535851485 & 1.41508910697179 \\
1 & 1.54841943801909 & 1.42123527210442 \\
2 & 1.54840928210547 & 1.42124884162844 \\
3 & 1.54840925984781 & 1.42124887136843 \\
4 & 1.54840925979908 & 1.42124887143354 \\
5 & 1.54840925979861 & 1.42124887143418 \\
\hline
\end{tabular}

limiting behaviour is illustrated with tests performed on the interval [0,30] discretized with $N=2000$ points). It is conjectured that all the excited states will converge to the fundamental solution $\left(u_{0}, v_{0}\right)$ when $\rho$ tends to 1 . Note that in this case, it becomes difficult for our method to capture excited solutions. Let us mention that all these computations can be made when dealing with solutions such that $u(0)=0, v(0)=\beta, u^{\prime}(0)=\gamma$ and $v^{\prime}(0)=0$. In this case, the necessary condition in order to have localized solutions now writes $\gamma^{2}=\rho \beta^{2}$.

\subsection{Higher-dimensional bright solitons}

We now investigate the determination of radial stationary solution of (3.2) in any space dimension. The new system writes

$$
\begin{cases}u^{\prime \prime}(r)+\frac{d-1}{r} u^{\prime}(r)-u(r)+u(r) v(r)=0 & r>0 \\ v^{\prime \prime}(r)+\frac{d-1}{r} v^{\prime}(r)-\rho v(r)+\frac{1}{2} u^{2}(r)=0 & r>0 \\ u(0)=\alpha, \quad v(0)=\beta, \quad u^{\prime}(0)=0, \quad v^{\prime}(0)=0 . & \end{cases}
$$

Unlike (3.3), it is not possible to find for localized solutions of (3.5) a relation between $\alpha$ and $\beta$, because of the frictional terms $u^{\prime} / r$ and $v^{\prime} / r$ that come into play if $d \geq 2$. We then use a continuation method that enables to 


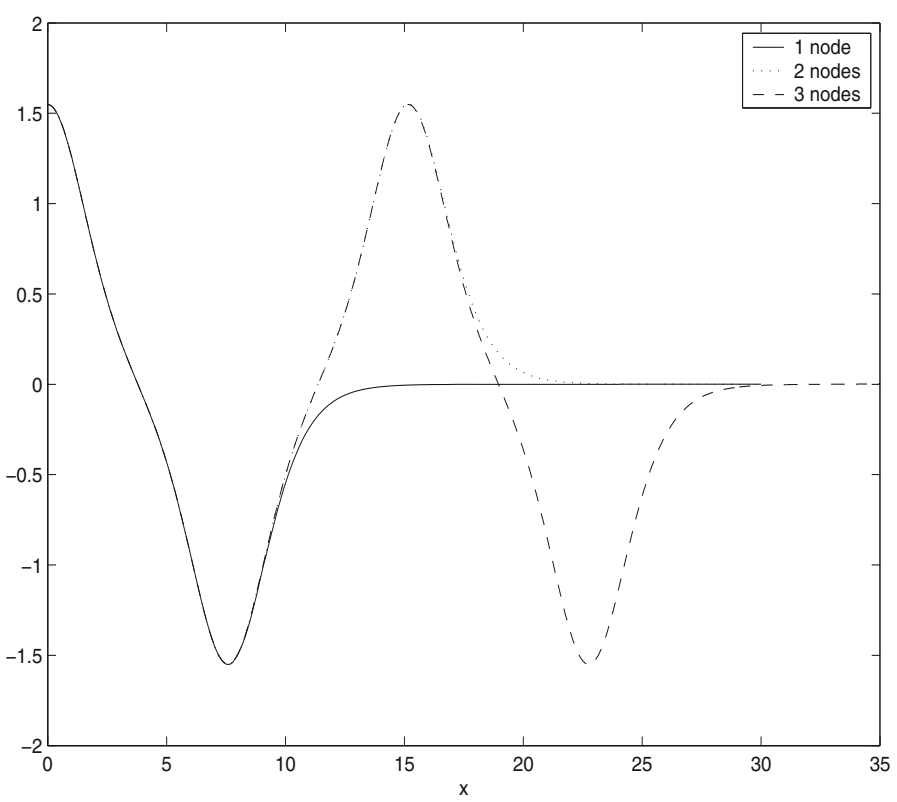

Figure 26. Plot of $u_{k}, \rho=0.5, k=1, k=2$ and $k=3$.

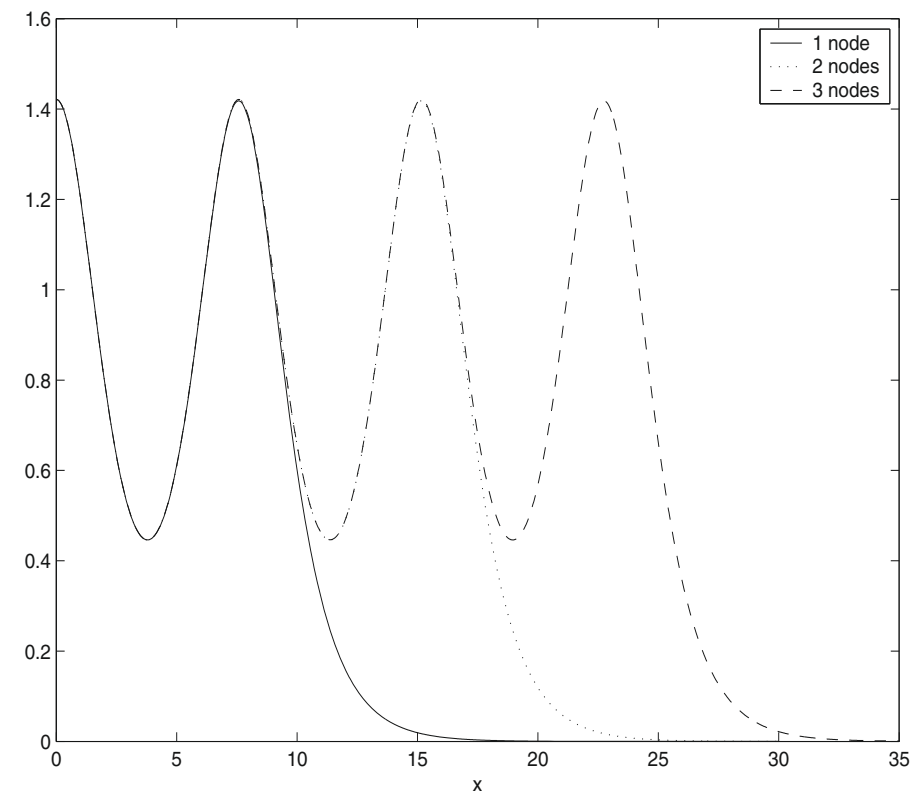

Figure 27. Plot of $v_{k}, \rho=0.5, k=1, k=2$ and $k=3$.

follow a path between one-dimensional solitons and $d$-dimensional solitons. Let us define $\left(u_{s}, v_{s}\right)$ as a spatially localized solution of (3.5) where $s$ is regarded as a continuous parameter that varies between 1 and $d$. Since it is possible to evaluate numerically any excited one-dimensional soliton (see Sect. 3.1), we assume that the starting point, say $\left(u_{1}, v_{1}\right)$, is known. We now follow the path between $\left(u_{1}, v_{1}\right)$ and the desired solution $\left(u_{d}, v_{d}\right)$, 


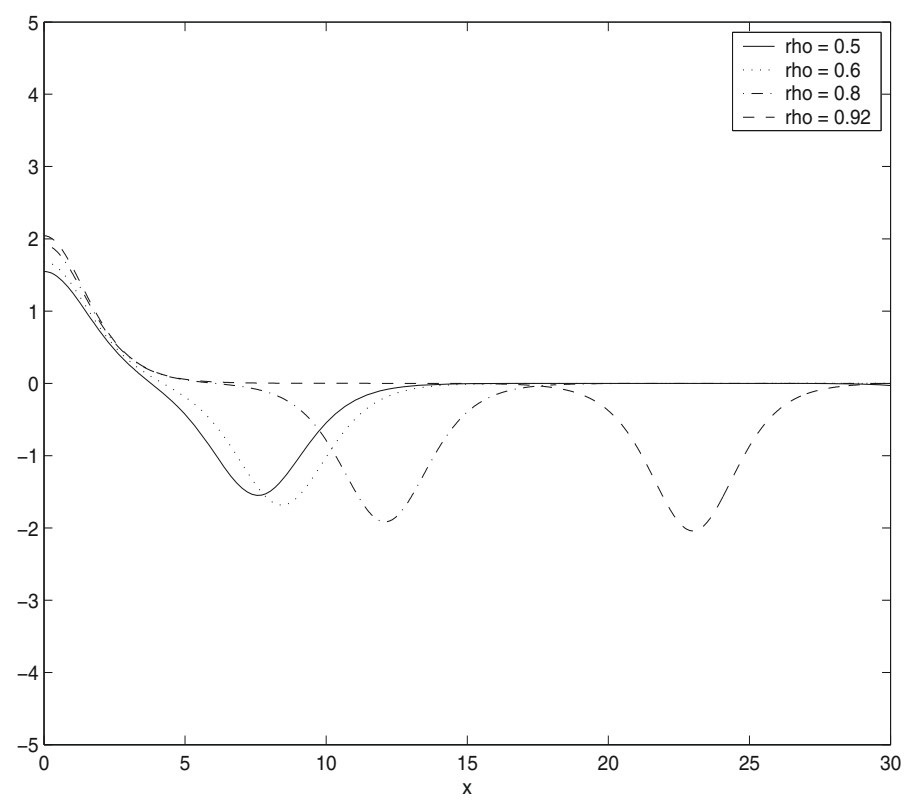

Figure 28. Plot of $u_{1}, \rho=0.7, \rho=0.8, \rho=0.9, \rho=0.92$.

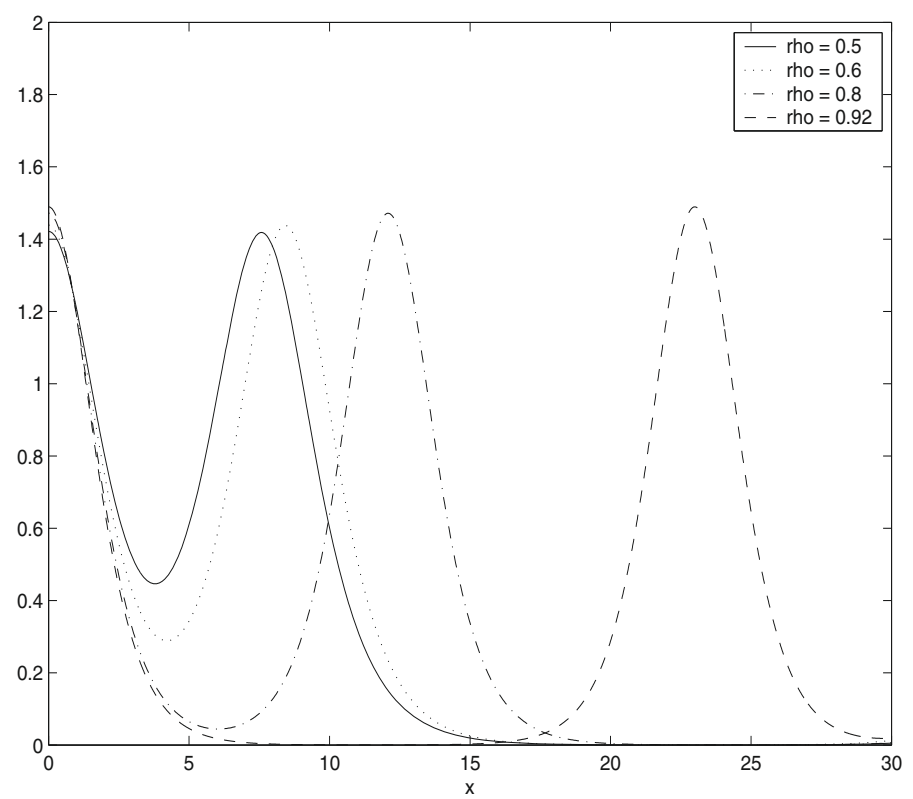

Figure 29. Plot of $v_{1}, \rho=0.7, \rho=0.8, \rho=0.9, \rho=0.92$.

expressing that for each $s,\left(u_{s}, v_{s}\right)$ satisfies the system

$$
\begin{cases}u_{s}^{\prime \prime}(r)+\frac{s-1}{r} u_{s}^{\prime}(r)-u_{s}(r)+u_{s}(r) v_{s}(r)=0 & r>0 \\ v_{s}^{\prime \prime}(r)+\frac{s-1}{r} v_{s}^{\prime}(r)-\rho v_{s}(r)+\frac{1}{2} u_{s}^{2}(r)=0 & r>0 .\end{cases}
$$


It implies that the derivative $\left(\dot{u}_{s}, \dot{v}_{s}\right)$ with respect to $s$ identically vanishes on this path. We thus get for each $r>0$ the identities

$$
\left\{\begin{array}{l}
\dot{u}_{s}^{\prime \prime}(r)+\frac{s-1}{r} \dot{u}_{s}^{\prime}(r)-\dot{u}_{s}(r)+v_{s}(r) \dot{u}_{s}(r)+u_{s}(r) \dot{v}_{s}(r)=-\frac{1}{r} u_{s}^{\prime}(r) \\
\dot{v}_{s}^{\prime \prime}(r)+\frac{s-1}{r} \dot{v}_{s}^{\prime}(r)-\rho \dot{v}_{s}(r)+u_{s}(r) \dot{u}_{s}(r)=-\frac{1}{r} v_{s}^{\prime}(r) .
\end{array}\right.
$$

This means that for each $s,\left(\dot{u}_{s}, \dot{v}_{s}\right)$ can be obtained from $\left(u_{s}, v_{s}\right)$ solving a linear nonhomogeneous elliptic-like system (it is not elliptic in the usual sense because $s$ is not an integer) that is noted $\left(\dot{u}_{s}, \dot{v}_{s}\right)=F\left(\left(u_{s}, v_{s}\right)\right)$. In order to compute $\left(u_{d}, v_{d}\right)$ starting from the initial data $\left(u_{1}, v_{1}\right)$, we thus have to solve the differential problem

$$
\left\{\begin{array}{l}
\left(\dot{u}_{s}, \dot{v}_{s}\right)=F\left(\left(u_{s}, v_{s}\right)\right), \quad s \in[1, d] \\
\left.\left(u_{s}, v_{s}\right)\right|_{s=1}=\left(u_{1}, v_{1}\right)
\end{array}\right.
$$

which can be easily done, again with a standard Runge-Kutta routine. Since radial exponentially decaying states are seeked, we consider the numerical domain $\left[0, r_{\max }\right]$ where this elliptic system is discretized on a uniform grid with spatial mesh $h$. Unfortunately, we have to deal with boundary condition at the right end-point of the segment in order to preserve the decreasing rate at infinity. Recalling that we drop off the nonlinear terms in order to find the correct asymptotics, we first have

$$
u(r) \sim C \frac{\mathrm{e}^{-r}}{r^{(d-1) / 2}} \quad \text { and } \quad v(r) \sim C \frac{\mathrm{e}^{-\sqrt{\rho} r}}{r^{(d-1) / 2}} .
$$

Differentiating with respect to $r$, we find that if $r$ is large enough,

$$
\frac{\partial u}{\partial r}+\left(1+\frac{d-1}{2 r}\right) u=0 \quad \text { and } \quad \frac{\partial v}{\partial r}+\left(\sqrt{\rho}+\frac{d-1}{2 r}\right) v=0
$$

Note that these conditions could be interpreted as transparent conditions for the linear part of the timedependent system rewritten in Laplace transform $\mathcal{L}$. In this case, $\mathcal{L}(\partial \psi / \partial t)=\omega \mathcal{L} \psi$ that can be compared with the zero-order term $\rho v$ and we find in (3.10) the pseudodifferential half-derivative operator of symbol $\sqrt{\omega}$ that is well-known in the theory of transparent conditions; see for example [4]. If $s$ once again stands for the continuous dimension parameter, then we have that for each $s \in[1, d]$

$$
\frac{\partial u_{s}}{\partial r}+\left(1+\frac{s-1}{2 r}\right) u_{s}=0 \quad \text { and } \quad \frac{\partial v_{s}}{\partial r}+\left(\sqrt{\rho}+\frac{s-1}{2 r}\right) v_{s}=0
$$

Now, we have to find correct boundary conditions for $\dot{u}_{s}$ and $\dot{v}_{s}$, since (3.8) will involve first-order derivatives. Differentiating with respect to $s$, the original boundary conditions lead us to

$$
\frac{\partial \dot{u}_{s}}{\partial r}+\left(1+\frac{s-1}{2 r}\right) \dot{u}_{s}=-\frac{1}{2 r} u_{s} \quad \text { and } \quad \frac{\partial \dot{v}_{s}}{\partial r}+\left(\sqrt{\rho}+\frac{s-1}{2 r}\right) \dot{v}_{s}=-\frac{1}{2 r} v_{s} .
$$

These boundary conditions guarantee that the computed solutions keep a correct asymptotics at infinity. However, the Cauchy data that is obtained in the one-dimensional case with the shooting method is such that $u_{1}$ changes its sign at $r=r_{\max }$, which is not consistent with (3.9). We thus decide to compute one-dimensional solitons on a spatial domain $\left[0, r_{\max }+r^{*}\right]$ and to restrict ourselves to the truncated domain $\left[0, r_{\max }\right]$ for the continuation method. In all the tests that have been made, the choice $r^{*}=8$ has given a correct starting point. In this case, boundary conditions (3.11) enable to preserve the right asymptotics for the solutions between 1 and $d$. Concerning now the left end-point of the numerical domain, differentiating the Neumann conditions $u_{s}^{\prime}(0)=v_{s}^{\prime}(0)=0$ with respect to $s$ simply gives us again $\dot{u}_{s}^{\prime}(0)=\dot{v}_{s}^{\prime}(0)=0$. One can refer to Appendix for the discretization of (3.7) and (3.11) that allows us to compute the discrete values of $\left(\dot{u}_{s}, \dot{v}_{s}\right)$ from $\left(u_{s}, v_{s}\right)$. 


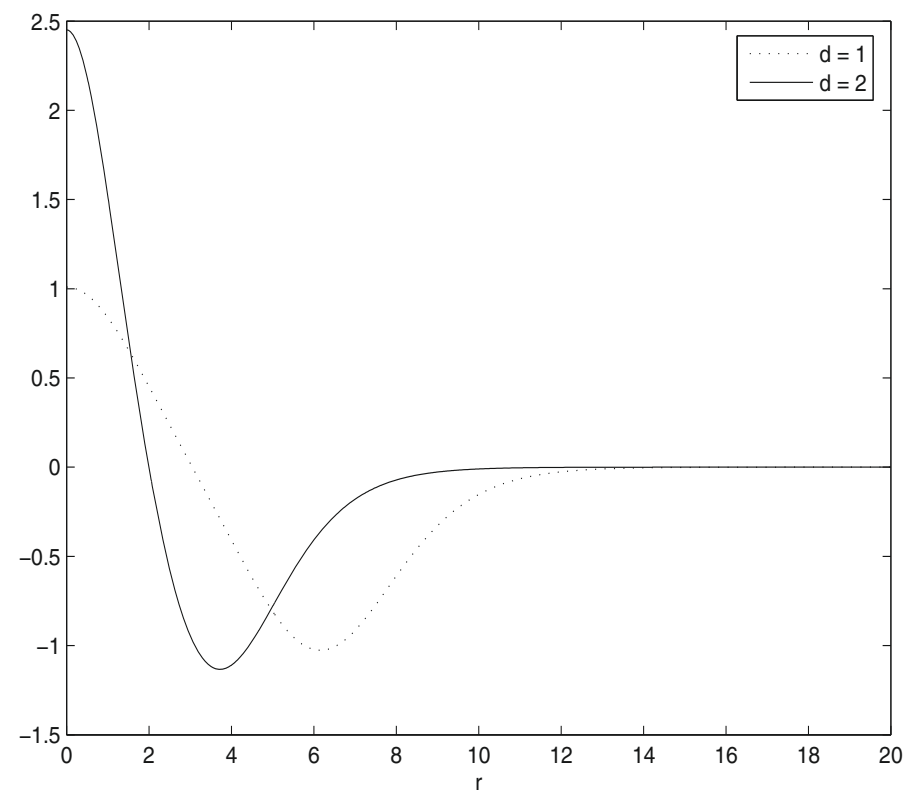

Figure 30. Plot of $u_{1}$, one-dimensional starting point and two-dimensional excited state, $\rho=0.2$.

We first plot the stationary states $\left(u_{1}, v_{1}\right)$ comparing them with the profiles obtained in the one-dimensional case, taken as starting points. We have used the following values of parameters: $\rho=0.2, N_{d}=100$ discretization points for the numerical resolution of (3.8) and $r_{\max }=20$. As seen in Figures 30 and 31, the one-dimensional solitons have traveled through the continuous path and have given radial excited two-dimensional profiles. Note that in [11], a continuation method has been used starting from one-dimensional explicit solutions with no nodes that can be calculated for $\rho=1$.

In Figures 32 and 33 are shown a collection of excited states computed in the case $d=2$ (when dealing with large number of nodes, the spatial domain has been enhanced as for solitons computed in Sect. 2). As for the scalar NLS equation, it is noted that the values of $u_{k}$ and $v_{k}$ at the origin is an increasing function of $k$. In Figure 33, we observe that $v_{1}$ is strictly decreasing in $\mathbb{R}^{+}$although the starting point admits two local extrema outside the origin. Surprisingly, for larger values of $k$, the last local extrema for $v_{k}$ in $1 d$ will persist as local extrema of $v_{k}$ in $2 d$, whereas the first ones close to the origin will be lost: as an example, $v_{2}$ (respectively $v_{3}$ ) only admits one local minimum and one local maximum (outside the origin) and has been calculated from a one-dimensional profile with 2 (respectively 3 ) local minimum and local maximum (outside the origin).

\subsection{Vortex solitons in two-dimensional case}

As for NLS equation, it is possible to look for solutions with dependence with respect to angular parameter $\theta$. We thus look for solutions of the form $u(r) \exp (\mathrm{i} m \theta)$ and $u(r) \exp (2 \mathrm{i} m \theta)$; the system to be solved now reads

$$
\left\{\begin{array}{l}
u^{\prime \prime}(r)+\frac{1}{r} u^{\prime}(r)-\frac{m^{2}}{r^{2}} u(r)-u(r)+\bar{u}(r) v(r)=0 \\
v^{\prime \prime}(r)+\frac{1}{r} v^{\prime}(r)-\frac{4 m^{2}}{r^{2}} v(r)-\rho v(r)+\frac{1}{2} u^{2}(r)=0 .
\end{array}\right.
$$




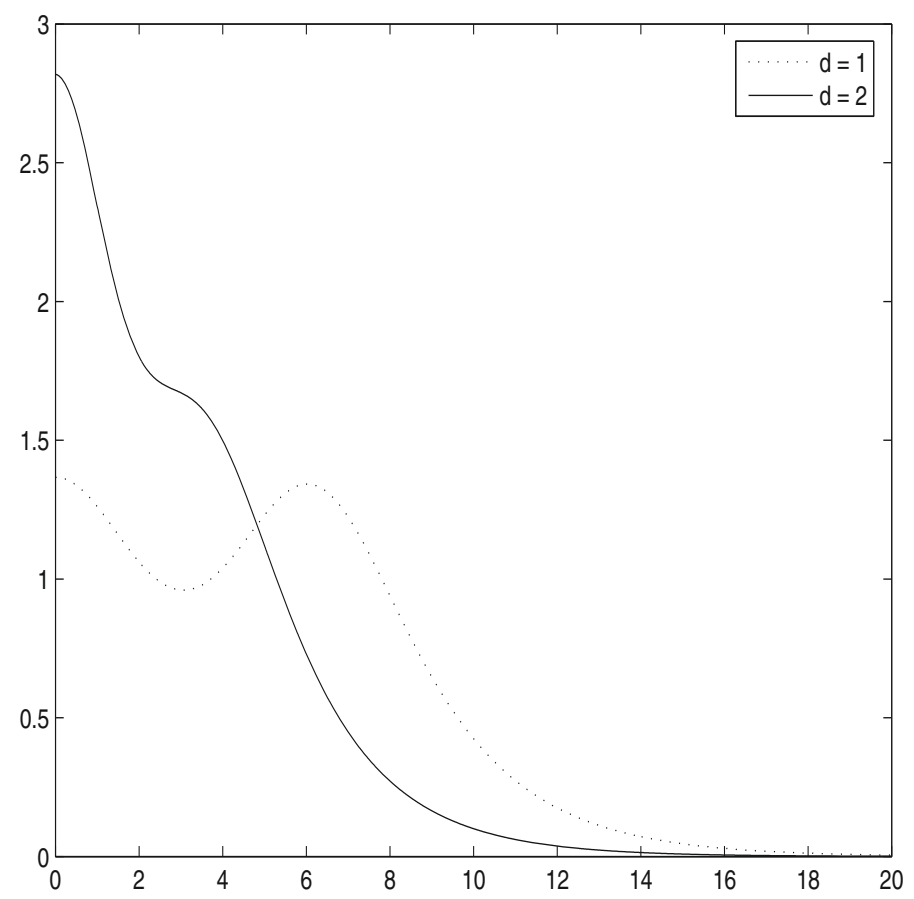

FIGURE 31. Plot of $v_{1}$, one-dimensional starting point and two-dimensional excited state, $\rho=0.2$.

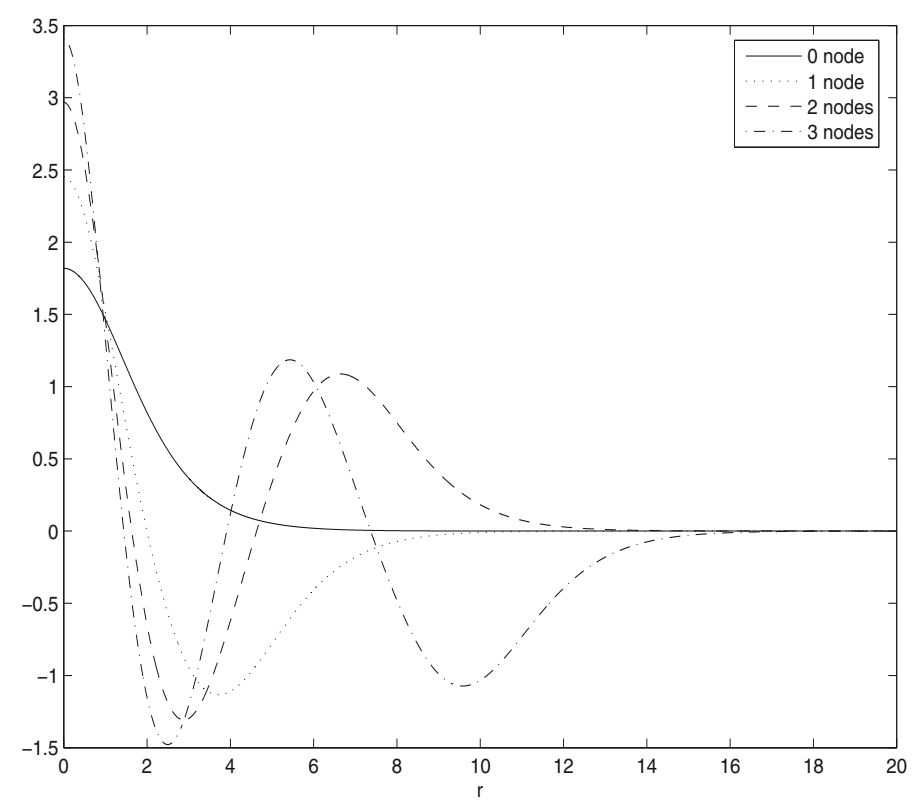

Figure 32. Plot of $u_{k}, \rho=0.5, k=0, k=1, k=2$ and $k=3$. 


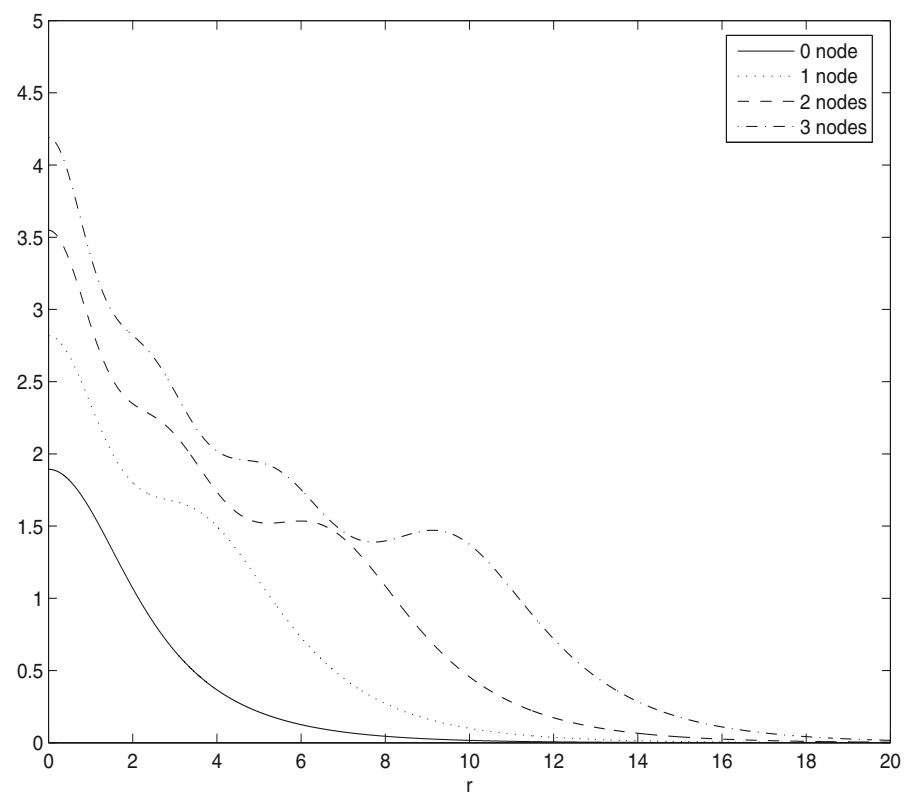

Figure 33. Plot of $v_{k}, \rho=0.2, k=0, k=1, k=2$ and $k=3$.

Such solutions vanish at $r=0$ and we set $u(r)=r^{m} U(r)$ and $v(r)=r^{2 m} V(r)$. The system turns into

$$
\left\{\begin{array}{l}
U^{\prime \prime}(r)+\frac{1+2 m}{r} U^{\prime}(r)-U(r)+r^{2 m} U(r) V(r)=0 \\
V^{\prime \prime}(r)+\frac{1+4 m}{r} V^{\prime}(r)-\rho V(r)+\frac{1}{2} U^{2}(r)=0 .
\end{array}\right.
$$

We decide to use the continuation procedure described above, taking $m$ as a continuation parameter that is still noted $s$ : we define the pair $\left(U_{s}(r), V_{s}(r)\right)$ as the solutions of (3.13). Differentiating with respect to $s \in[0, m]$, we get

$$
\left\{\begin{array}{r}
\dot{U}_{s}^{\prime \prime}(r)+\frac{1+2 s}{r} \dot{U}_{s}^{\prime}(r)-\dot{U}_{s}(r)+r^{2 s} V_{s}(r) \dot{U}_{s}(r)+r^{2 s} U_{s}(r) \dot{V}_{s}(r)= \\
-\frac{2}{r} U_{s}(r)-2 \log r r^{2 s} U_{s}(r) V_{s}(r) \\
\dot{V}_{s}^{\prime \prime}(r)+\frac{1+4 s}{r} \dot{V}_{s}^{\prime}(r)-\rho \dot{V}_{s}(r)+U_{s}(r) \dot{U}_{s}(r)=-\frac{4}{r} V_{s}(r) .
\end{array}\right.
$$

If $\left(U_{s}, V_{s}\right)$ is known, this gives us $\left(\dot{U}_{s}, \dot{V}_{s}\right)$. Here again, a differential solver enables us to compute $\left(U_{m}, V_{m}\right)$ from Cauchy data $\left(U_{0}, V_{0}\right)$ which is nothing else but the bright solitons in the two-dimensional case, because of the the friction terms $U^{\prime}(r) / r$ and $V^{\prime}(r) / r$ in the system at $s=0$. The boundary conditions set at the right side of the numerical domain are the same as for bright solitons. We show in Figures 34 and 35 the profiles of excited states $\left(u_{k, m}, v_{k, m}\right)$ obtained in the case $m=1$ for various $k$.

Taking larger values of $m$ has faced us with a stability problem, due to the fact that the original solutions are $(u(r), v(r))=\left(r^{m} U(r), r^{2 m} V(r)\right)$. Consequently, a small perturbation in the computation of $(U, V)$ will generate a catastrophic variation on $u$ and $v$ at large $r$. This can be dramatic when seeking excited states with large number of nodes. If $m>1$, we then use the previous continuation method from 0 to 1 and we go back to 


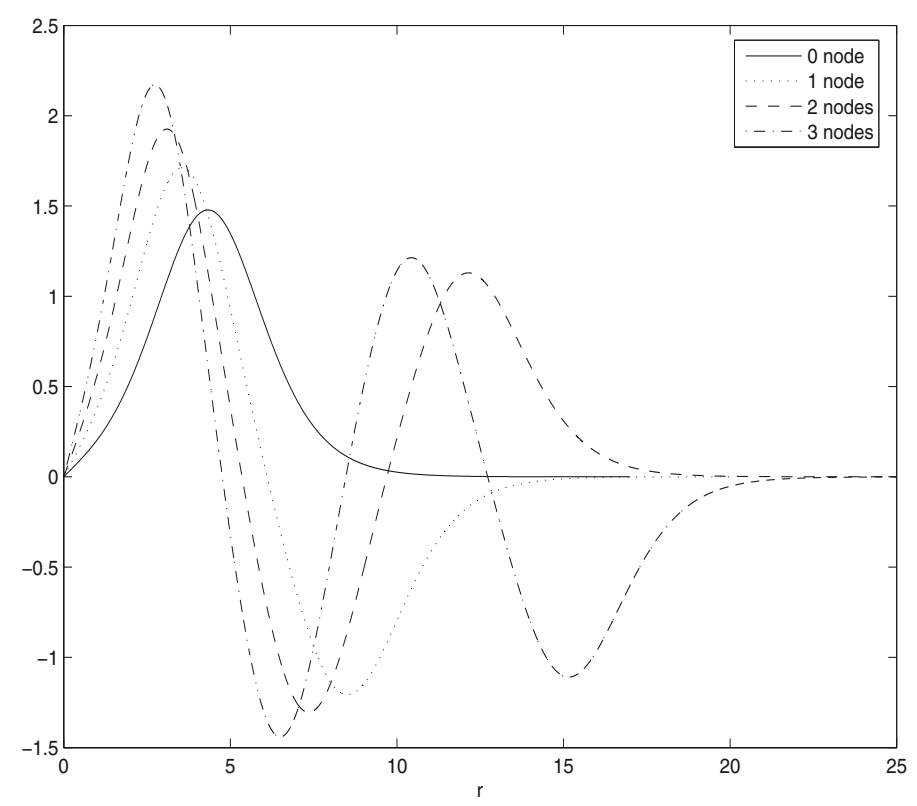

Figure 34. Plot of $u_{k, m}, m=1, \rho=0.2, k=0, k=1, k=2$ and $k=3$.

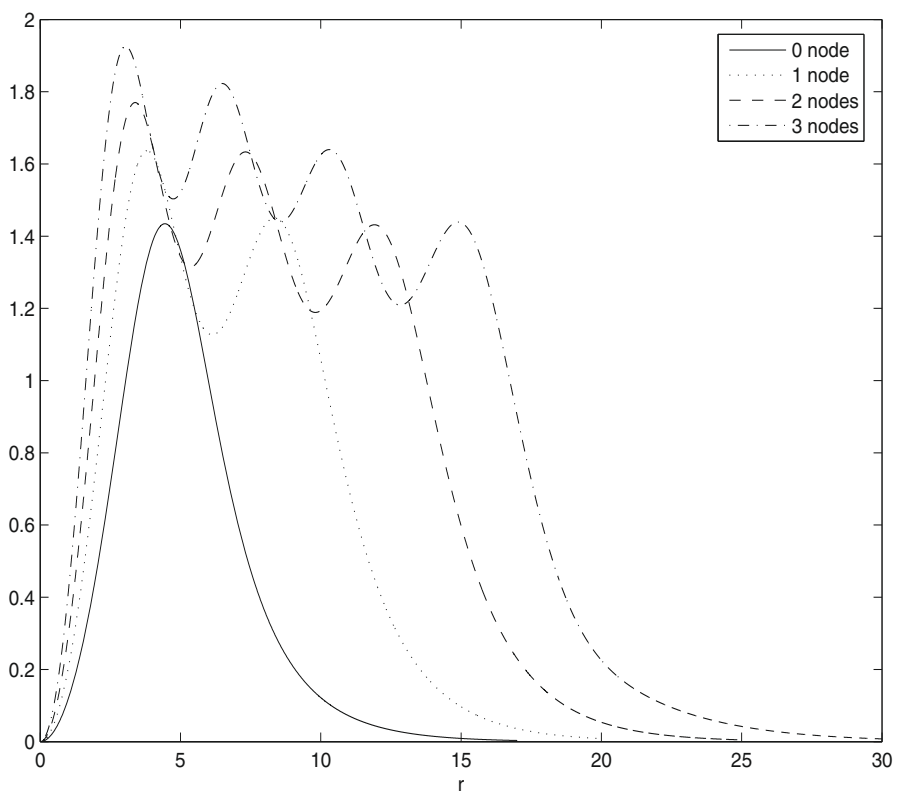

Figure 35. Plot of $v_{k, m}, m=1, \rho=0.2, k=0, k=1, k=2$ and $k=3$.

the initial system from 1 to $m$; the system that is solved in order to compute $(\dot{u}, \dot{v})$ is now

$$
\left\{\begin{array}{l}
\dot{u}_{s}^{\prime \prime}(r)+\frac{1}{r} \dot{u}_{s}^{\prime}(r)-\frac{s^{2}}{r^{2}} \dot{u}_{s}(r)-\dot{u}_{s}(r)+v_{s}(r) \dot{u}_{s}(r)+u_{s}(r) \dot{v}_{s}(r)=\frac{2 s}{r^{2}} u_{s}(r) \\
\dot{v}_{s}^{\prime \prime}(r)+\frac{1}{r} \dot{v}_{s}^{\prime}(r)-\frac{4 s^{2}}{r^{2}} \dot{v}_{s}(r)-\rho \dot{v}_{d}(r)+u_{s}(r) \dot{u}_{s}(r)=\frac{8 s}{r^{2}} v_{s}(r) .
\end{array}\right.
$$




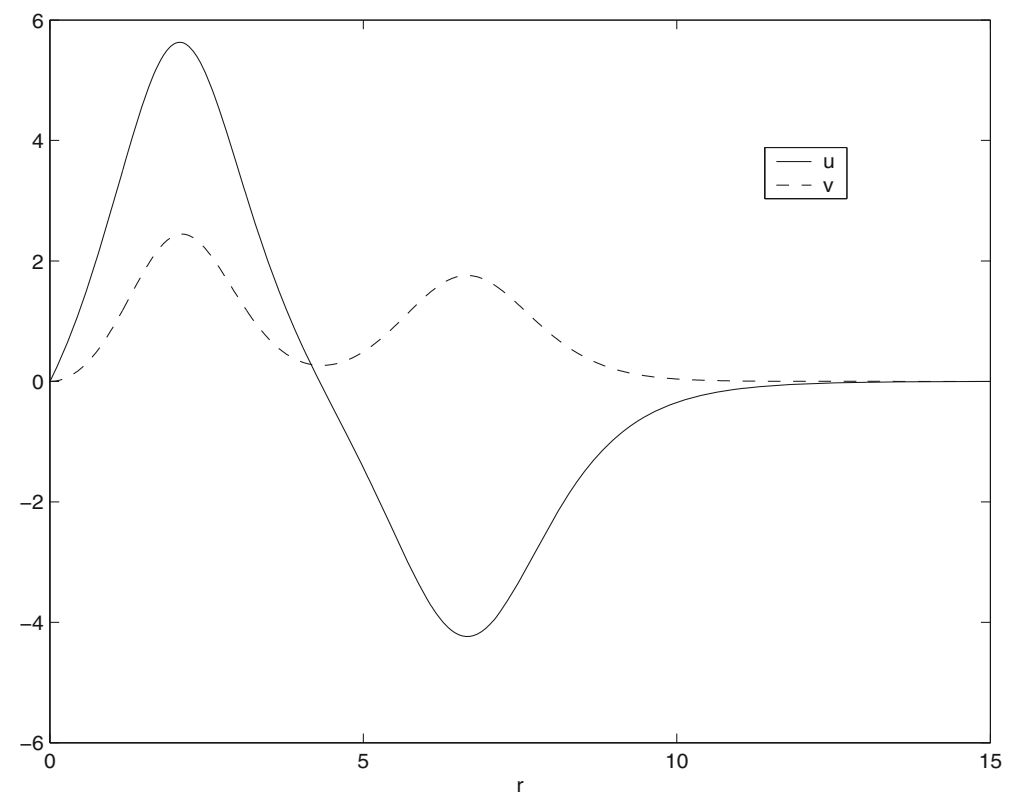

Figure 36. Plot of $u_{1,1}$ and $v_{1,1}, \rho=4$.

In this case, the two continuation techniques have been compared and the solutions that are found are quite the same. As for equation (1.1), it seems that taking larger values of $m$ translates the vortex.

We have also used this strategy with respect to parameter $\rho$, between its initial value and some final value $\rho_{f}$. Indeed, it had been observed that excited bright solitons for the one-dimensional case do not exist if $\rho \geq 1$. We then decided to investigate if excited vortex states could exist anyway for large $\rho$. Continuation technique enabled us to compute excited states for $\rho>1$ (that is for $\rho_{f}>1$ ) (see Fig. 36) where the first excited vortex solution has been computed for $\rho=4$ ). This possibility to find vortex solutions for large $\rho$ points out that the continuation techniques that are successively used are not commutative: it would be impossible to reach vortex excited states at some $\rho \geq 1$ starting from one-dimensional excited bright solitons computed with $\rho$ since these states do not exist. Note that in [6], vortex states have been computed in the case $k=0$ with a finite-difference strategy.

\section{THREE-WAVE MODELS}

We finally investigate the case where the nonlinear model involves 3 coupled waves noted $u, v$ and $w$, respectively associated to carrying wave numbers $k_{u}, k_{v}$ and $k_{w}$, with the matching condition $k_{w}=k_{u}+k_{v}$. The system that has to be solved writes now

$$
\left\{\begin{array}{c}
\Delta u-u+\bar{v} w=0 \\
\Delta v-\gamma v+\bar{u} w=0 \\
\Delta w-\rho w+u v=0
\end{array}\right.
$$

where $\rho$ and $\gamma$ are strictly positive constants. If $\gamma=1$, it is possible to find real solutions of the form $(u, u, v)$, where $u$ and $v$ solve the two-wave model described in the previous section. 


\subsection{One-dimensional solitons}

For $\gamma \neq 1$, it becomes impossible to use a scalar shooting strategy as before to compute stationary states. This can be explained by the relation that can be obtained between the values $\alpha, \beta$ and $\delta$ of $u, v$ and $w$ at $r=0$ (assuming that the expected solutions of $(4.1)$ satisfy $\left.u^{\prime}(0)=v^{\prime}(0)=w^{\prime}(0)=0\right)$. Indeed, following the strategy used for Proposition 3.1, it is possible to show that the new relation reads now

$$
\alpha^{2}+\gamma \beta^{2}+\rho \delta^{2}-2 \alpha \beta \delta=0
$$

Thus, it becomes impossible to express all the values at the origin with respect to one single parameter that can be used as a shooting parameter. We then intend to use a continuation technique, starting from the two-wave system obtained initially setting $\gamma=1, u_{1}=v_{1}=u$ and $w_{1}=v$ that can be computed using the algorithm described in Section 3.

Let $\left(u_{s}, v_{s}, w_{s}\right)$ be the solution of the nonlinear system

$$
\left\{\begin{array}{c}
u_{s}^{\prime \prime}(r)-u_{s}(r)+w_{s}(r) v_{s}(r)=0 \\
v_{s}^{\prime \prime}(r)-s v_{s}(r)+w_{s}(r) u_{s}(r)=0 \\
w_{s}^{\prime \prime}(r)-\rho w_{s}(r)+u_{s}(r) v_{s}(r)=0
\end{array}\right.
$$

We assume that the one-parameter family $\left(u_{s}, v_{s}, w_{s}\right)$ solves (4.3) for each $s$, then if we differentiate with respect to $s$, we find that

$$
\left\{\begin{array}{l}
\dot{u}_{s}^{\prime \prime}(r)-\dot{u}_{s}(r)+w_{s}(r) \dot{v}_{s}(r)+v_{s}(r) \dot{w}_{s}(r)=0 \\
\dot{v}_{s}^{\prime \prime}(r)-s \dot{v}_{s}(r)+w_{s}(r) \dot{u}_{s}(r)+u_{s}(r) \dot{w}_{s}(r)=v_{s}(r) \\
\dot{w}_{s}^{\prime \prime}(r)-\rho \dot{w}_{s}(r)+v_{s}(r) \dot{u}_{s}(r)+u_{s}(r) \dot{v}_{s}(r)=0
\end{array}\right.
$$

for each $s$ between 1 and $\gamma$. This system enables to find $\left(\dot{u}_{s}, \dot{v}_{s}, \dot{w}_{s}\right)$ if $\left(u_{s}, v_{s}, w_{s}\right)$ is known, under the general form $\left(\dot{u}_{s}, \dot{v}_{s}, \dot{w}_{s}\right)=G\left(\left(u_{s}, v_{s}, w_{s}\right)\right)$. As in the two-wave model, $\left(\dot{u}_{s}, \dot{v}_{s}, \dot{w}_{s}\right)$ solves a linear system with coefficients and source term depending on $\left(u_{s}, v_{s}, w_{s}\right)$. We then solve the differential problem

$$
\left\{\begin{array}{l}
\left(\dot{u}_{s}, \dot{v}_{s}, \dot{w}_{s}\right)=G\left(\left(u_{s}, v_{s}, w_{s}\right)\right), \quad s \in I \\
\left.\left(u_{s}, v_{s}, w_{s}\right)\right|_{s=1}=\left(u_{1}, u_{1}, v_{1}\right)
\end{array}\right.
$$

where $I=[\gamma, 1]$ if $\gamma<1$ and $I=[1, \gamma]$ if $\gamma \geq 1$ and find the three-wave soliton starting from the two-wave soliton. Note that in this case, the starting point is such that $\left.u_{s}\right|_{s=1}=\left.v_{s}\right|_{s=1}$ and initializing with an excited state will lead us to a three-wave excited state with the same number of nodes for $u$ and $v$. In order to solve the system at each $s$, we add the following boundary conditions at $r=r_{\max }$, following the strategy defined for the two-wave system:

$$
\frac{\partial \dot{u}_{s}}{\partial r}+\dot{u}_{s}=0, \frac{\partial \dot{v}_{s}}{\partial r}+\sqrt{s} \dot{v}_{s}=-\frac{1}{2 \sqrt{s}} v_{s} \text { and } \frac{\partial \dot{w}_{s}}{\partial r}+\sqrt{\rho} \dot{w}_{s}=0
$$

In Figure 37 is plotted the first component $u$ of the solutions obtained for $\gamma=1,2,3$ and 4 , computed for $\rho=0.5$ on the numerical domain $[0,15]$ discretized with 1000 spatial points, starting with the two-wave solution $\left(u_{0}, v_{0}\right)$. In this case, $(4.5)$ has been solved using $N_{\gamma}=40$ discretization points in $\gamma$. Other computations have shown that the state $\left(u_{0}, v_{0}, w_{0}\right)$ seems to exist for each $\gamma>0$. The solution at final value $\gamma$ is such that relation (4.2) is satisfied.

The same tests have been made with $\left(u_{1}, v_{1}\right)$ as the starting point (see Fig. 38 the plots of $u_{1}$ computed with different values of $\gamma)$ : in this case, we notice that the final solution keeps the same structure $\left(u_{1}\right.$ and $v_{1}$ have one node and $w_{1}$ remains strictly positive). It is remarkable that $u_{1}$ and $v_{1}$ vanish at the same point; 


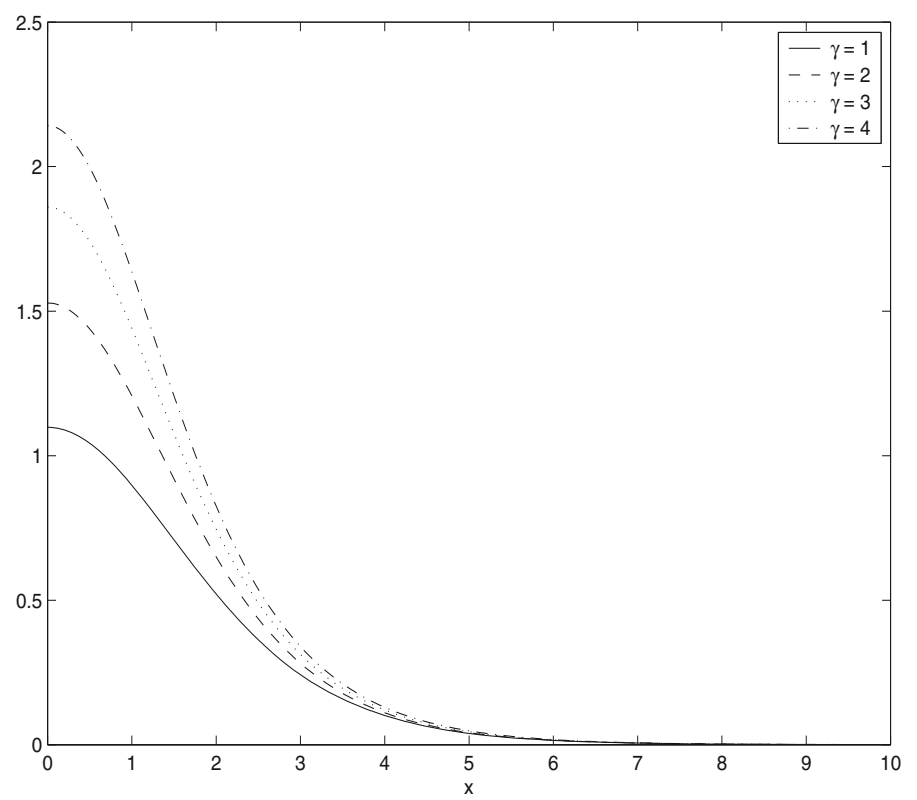

Figure 37. Plot of $u_{0}, \rho=0.5, \gamma=1,2,3,4$.

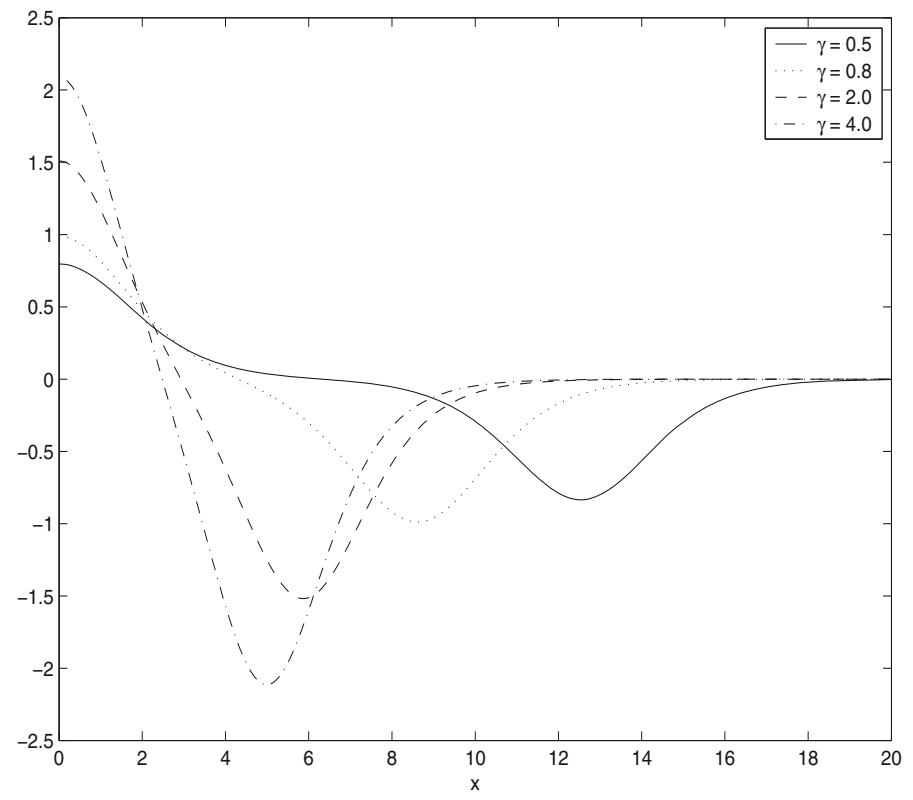

Figure 38. Plot of $u_{1}, \rho=0.5, \gamma=0.5,0.8,2,4$.

this implies by the maximum principle that $w_{1} \geq 0$. Furthermore, when $\gamma$ decreases, the local minimum of $w_{1}$ seems to tend to zero and the two extrema of $w_{1}$ are attained at some values of $r$ that become large. After this, it is observed that relation (4.2) is no more fulfilled and the computed solution cannot be considered as a correct one. More tests have shown that for each $k \geq 1$, there exists a threshold value $\gamma^{*}=\gamma^{*}(\rho, k)<1$ such that no solution $\left(u_{k}, v_{k}, w_{k}\right)$ with equal number of nodes for $u_{k}$ and $v_{k}$ will be found for $\gamma \leq \gamma^{*}$. This indicates to us 


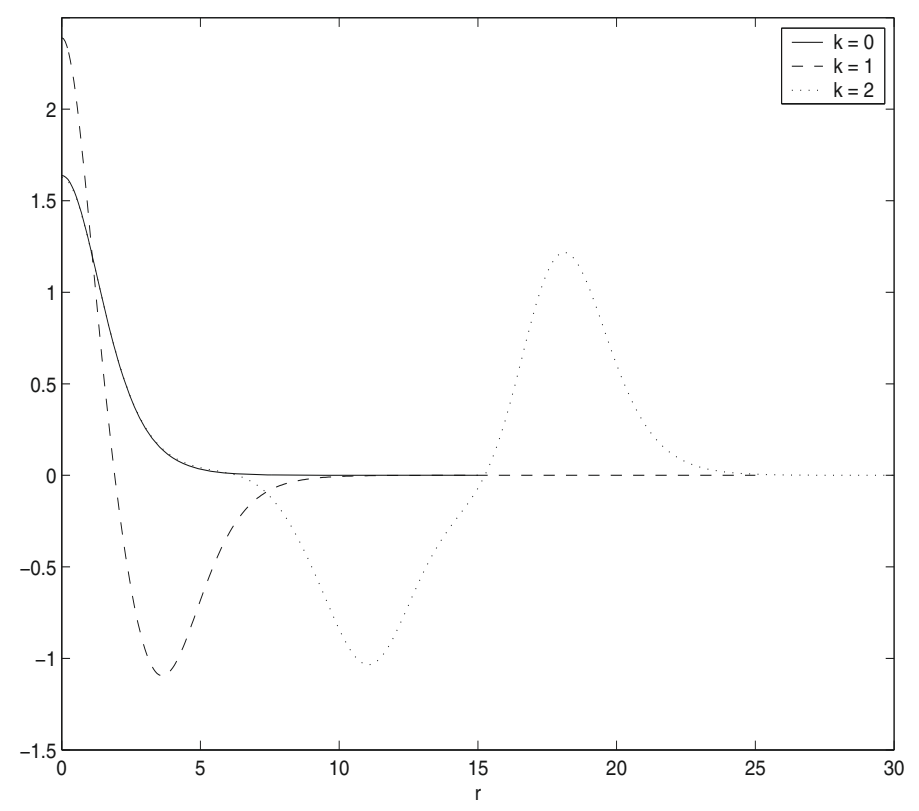

Figure 39. Plot of $u_{k}, \rho=0.5, \gamma=0.8, d=2, k=0,1,2$.

that three-wave $k$ th excited states could only exist for $\gamma \in] \gamma^{*}(\rho, k), \infty[$ which differs from the two-wave excited states where any excited solitons seem to exist for any $\rho<1$. If now $\gamma \geq 1>\gamma^{*}(\rho, k)$, excited solitons have be computed with $k$ arbitrarily large; furthermore, for each $k$, the local minima of $w_{k}$ increase with $\gamma$. Finally, it has been noticed that at $\rho$ prescribed smaller than $1,\left(\gamma^{*}(\rho, k)\right)_{k>1}$ is an increasing sequence. Of course, this investigation only concerns localized solutions with the same number of nodes for the first two components $u$ and $v$. Others kinds of stationary states may exist in this case.

\subsection{Generalizations}

The same method as in Section 3 can be used in order to find stationary states in dimension $d \geq 2$. In this new context, we compute the path between the three-wave starting point $\left(u_{1}, v_{1}, w_{1}\right)$ in one space dimension and the solution $\left(u_{d}, v_{d}, w_{d}\right)$ of (4.1) at dimension $d$ solving a differential system similar to the one described for two-wave system. We view in Figure 39 the first wave profile obtained in the two-dimensional case for $\rho=0.5$ and $\gamma=0.8$, starting from $\left(u_{k}, v_{k}, w_{k}\right)(0 \leq k \leq 2)$. As opposed to what was observed for the two-wave case, the solutions obtained for $k=2$ indicate that excited states with same number of nodes for $u$ and $v$ may not exist for large $k$ when $\gamma$ is prescribed: the two local extrema are reached at some $r_{0}$ and $r_{1}$ that go away from the origin. The conclusion is the same as in the one-dimensional case. If $\gamma>1$, it becomes again possible to compute excited states (see Fig. 40 where the first state $u$ has been plotted for computations made with $\gamma=1.5)$. This regime is very different qualitatively from the one found for $\gamma=0.8$.

Finally, the method still works for the determination of three-wave vortices solutions, that write $u\left(t, x_{1}, x_{2}\right)=$ $u(r) \exp \left(\mathrm{i} m_{u} \theta\right), v\left(t, x_{1}, x_{2}\right)=v(r) \exp \left(\mathrm{i} m_{v} \theta\right)$ and $w\left(t, x_{1}, x_{2}\right)=w(r) \exp \left(\mathrm{i}\left(m_{u}+m_{v}\right) \theta\right)$, where $m_{u}$ and $m_{v}$ are integers. Using the change of variables $u(r)=U(r) r^{m_{u}}, v(r)=V(r) r^{m_{v}}$ and $w(r)=W(r) r^{m_{u}+m_{v}}$, the system 


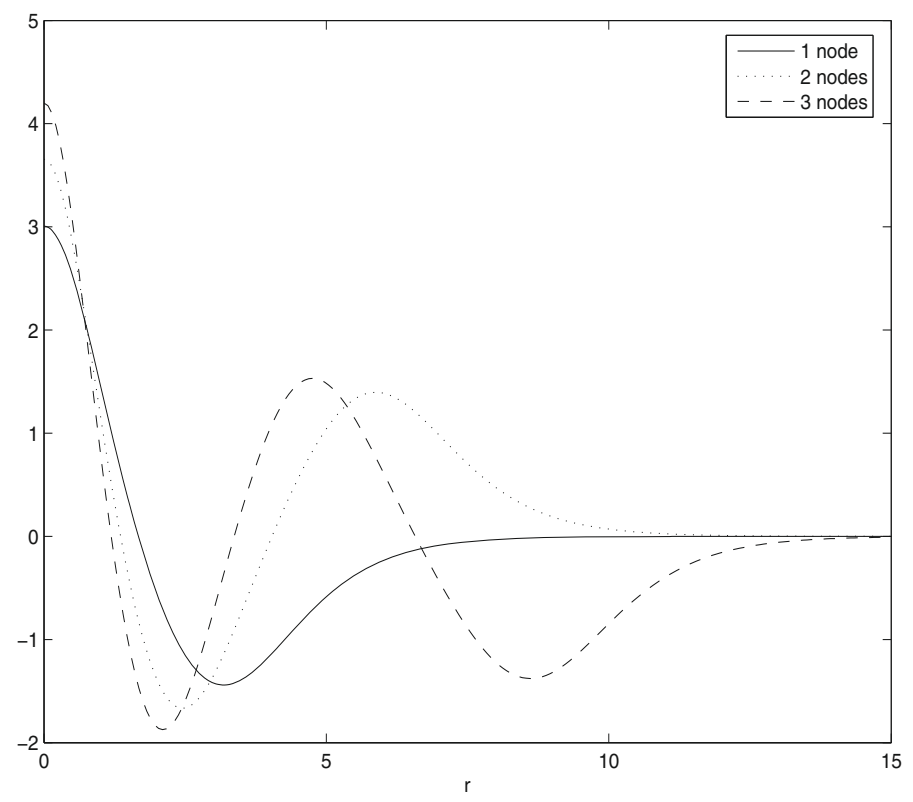

Figure 40. Plot of $u_{k}, \rho=0.5, \gamma=1.5, d=2, k=1,2,3$.

that has to be solved writes

$$
\left\{\begin{array}{l}
U^{\prime \prime}(r)+\frac{1+2 m_{u}}{r} U^{\prime}(r)-U(r)+r^{2 m_{v}} W(r) V(r)=0 \\
V^{\prime \prime}(r)+\frac{1+2 m_{v}}{r} V^{\prime}(r)-\gamma V(r)+r^{2 m_{u}} W(r) U(r)=0 \\
W^{\prime \prime}(r)+\frac{1+2\left(m_{u}+m_{v}\right)}{r} W^{\prime}(r)-\rho W(r)+U(r) V(r)=0 .
\end{array}\right.
$$

We solve this system using a continuation technique from a starting point given by the three-wave solitons $(u, v, w)$, that is solutions of (4.1) obtained in the two-dimensional case. Here, $m_{u}$ stands for the continuation parameter, setting $m_{v}=\theta m_{u}$. In other words, the continuation acts on both $m_{u}$ and $m_{v}$ and we initialize the system with $\left.\left(U_{s}, V_{s}, W_{s}\right)\right|_{s=0}=(u, v, w)$. Once again, the path between 0 and $m_{u}$ is calculated by solving a differential system and allows us to calculate $k$ th excited vortex noted $\left(u_{k, m_{u}, m_{v}}, v_{k, m_{u}, m_{v}}, w_{k, m_{u}, m_{v}}\right)$. This algorithm is of course also valid for the computation of special solutions for which $m_{v}+m_{v}=0$ and $m_{u} \neq 0$, meaning that $w$ does not vanish at $r=0: u$ and $v$ stand as vortices and $w$ reduces to a usual bright soliton.

\section{Conclusion}

In this paper, we wave computed solitons for equations arising in nonlinear optics. First, we have used the classical shooting method in order to find numerical approximations of the excited states of (1.1). This method is quite robust and it allowed us to study various asymptotics in several cases: when the number of zeros $k$ is large, it is observed that $\left\|u_{k}\right\|_{L^{\infty}},\left\|u_{k}\right\|_{L^{2}},\left\|\nabla u_{k}\right\|_{L^{2}}$ and $S\left(u_{k}\right)$ are governed by scaling laws in terms of $k$ with specific exponents. In the two-dimensional case, vortex solitons have been found and here again, scaling laws involving $\sigma$ and $m$ have been numerically found. In the case of negative $\sigma$ (only investigated for $\omega=\alpha=-1$ ), we have found that the power only depends on $k$. Finally, the case $\sigma \rightarrow \sigma^{*}$ has been considered at fixed number of zeros, leading us to the conclusion that the asymptotics depends on $k$. This points out that this asymptotic behaviour is not driven by a scaling argument that would give a uniform law in terms of the number of nodes. 
Then, we focused on the system of wave propagation in quadratic media. It has been shown that the shooting strategy still works in the case of two coupled waves. Even in the one-dimensional case, this model admits a large variety of stationary states (including excited states), as opposed to NLS equation. A continuation algorithm has been proposed in order to compute the path between one-dimension and $d$ dimensional solitons where the shooting method cannot be directly used. It is believed that excited states do not exist for any values of parameter $\rho$ in the system. In the two-dimensional case, vortex have been obtained with use of a change of variable that transforms the desired solutions into structures that do not vanish at the origin. These solutions are found to exist for a wider range of parameters as for bright solitons.

We have also been able to compute three-wave solitons using the continuation method, starting from two-wave solutions, as well as vortex solutions. Here again, we conjecture that depending on parameters $\rho$ and $\gamma$, this model does not possess an infinite number of excited localized solutions. It is remarkable that one-dimensional solitons for the two-wave system can be extended in such stable way in order to compute solitons for other equations: higher dimensional cases, vortex solitons, different parameters in the system, etc. This kind of method could be used in other contexts where stationary solutions cannot be explicitly integrated as solutions of a scalar differential equation.

\section{Appendix}

We give now the linear system obtained when solving differential system (3.7) for the computation of solitons in the two-wave model in dimension $d$. We recall that in this case, $s$ denotes the parameter that continuously joins one-dimensional solitons and $d$ dimensional solitons. Let $r_{j}=j h$ for $0 \leq j \leq N$ and let $\left(u_{s}\right)_{j},\left(v_{s}\right)_{j},\left(\dot{u}_{s}\right)_{j}$ and $\left(\dot{u}_{s}\right)_{j}$ respectively stand for the approximate values of $u_{s}, v_{s}, \dot{u}_{s}$ and $\dot{v}_{s}$ at $r=r_{j}$.

- For $j=0$, we have to investigate the discretization of the singular term $\dot{u}_{s}^{\prime}(r) / r$. As seen in Section 2.1, we have $\dot{u}_{s}^{\prime}(r) / r \rightarrow \dot{u}_{s}^{\prime \prime}(0)$ as $r \rightarrow 0$. Since $\dot{u}_{s}^{\prime}(0)=0$, it follows that

$$
\left(\dot{u}_{s}\right)_{1}=\left(\dot{u}_{s}\right)_{0}+\frac{h^{2}}{2}\left(\dot{u}_{s}^{\prime \prime}\right)_{0}+\mathcal{O}\left(h^{3}\right)
$$

and the second derivative at $r=0$ is approximated by $2\left(\left(\dot{u}_{s}\right)_{1}-\left(\dot{u}_{s}\right)_{0}\right) / h^{2}$. Consequently, the first equation writes

whereas the second one is

$$
-\left(\dot{u}_{s}\right)_{0}+\frac{2 s}{h^{2}}\left(\left(\dot{u}_{s}\right)_{1}-\left(\dot{u}_{s}\right)_{0}\right)+\left(v_{s}\right)_{0}\left(\dot{u}_{s}\right)_{0}+\left(u_{s}\right)_{0}\left(\dot{v}_{s}\right)_{0}=\frac{2}{h^{2}}\left(\left(u_{s}\right)_{1}-\left(u_{s}\right)_{0}\right)
$$

$$
-\rho\left(\dot{v}_{s}\right)_{0}+\frac{2 s}{h^{2}}\left(\left(\dot{v}_{s}\right)_{1}-\left(\dot{v}_{s}\right)_{0}\right)+\left(u_{s}\right)_{0}\left(\dot{u}_{s}\right)_{0}=\frac{2}{h^{2}}\left(\left(v_{s}\right)_{1}-\left(v_{s}\right)_{0}\right) .
$$

- For $1 \leq j \leq N-1$, the discretization of (3.7) leads us to the discrete equations

$$
\begin{array}{r}
-\left(\dot{u}_{s}\right)_{j}+\frac{1}{h^{2}}\left(\left(\dot{u}_{s}\right)_{j+1}-2\left(\dot{u}_{s}\right)_{j}+\left(\dot{u}_{s}\right)_{j-1}\right)+\frac{s-1}{2 h r_{j}}\left(\left(\dot{u}_{s}\right)_{j+1}-\left(\dot{u}_{s}\right)_{j-1}\right) \\
+\left(v_{s}\right)_{j}\left(\dot{u}_{s}\right)_{j}+\left(u_{s}\right)_{j}\left(\dot{v}_{s}\right)_{j}=-\frac{1}{2 h r_{j}}\left(\left(u_{s}\right)_{j+1}-\left(u_{s}\right)_{j-1}\right) \\
-\left(\dot{v}_{s}\right)_{j}+\frac{1}{h^{2}}\left(\left(\dot{v}_{s}\right)_{j+1}-2\left(\dot{v}_{s}\right)_{j}+\left(\dot{v}_{s}\right)_{j-1}\right)+\frac{s-1}{2 h r_{j}}\left(\left(\dot{v}_{s}\right)_{j+1}-\left(\dot{v}_{s}\right)_{j-1}\right) \\
+\left(u_{s}\right)_{j}\left(\dot{u}_{s}\right)_{j}=-\frac{1}{2 h r_{j}}\left(\left(v_{s}\right)_{j+1}-\left(v_{s}\right)_{j-1}\right) .
\end{array}
$$

- In order to find the equation set at the last point $r_{N}$ of the domain, we start with the Taylor expansion

$$
\left(\dot{u}_{s}\right)_{N-1}=\left(\dot{u}_{s}\right)_{N}-h\left(\dot{u}_{s}^{\prime}\right)_{N}+\frac{h^{2}}{2}\left(\dot{u}_{s}^{\prime \prime}\right)_{N}+\mathcal{O}\left(h^{3}\right)
$$


from which we deduce the following relation, plugging the boundary condition $\left(\dot{u}_{s}^{\prime}\right)_{N}=-\left(1+(s-1) / 2 r_{N}\right)\left(\dot{u}_{s}\right)_{N}-$ $\left(u_{s}\right)_{N} / 2 r_{N}$ :

$$
\left(\dot{u}_{s}^{\prime \prime}\right)_{N}=\frac{2}{h^{2}}\left(\dot{u}_{s}\right)_{N-1}-\left[\frac{2}{h^{2}}+\frac{2}{h}\left(1+\frac{s-1}{2 r_{N}}\right)\right]\left(\dot{u}_{s}\right)_{N}-\frac{1}{h r_{N}}\left(u_{s}\right)_{N}+\mathcal{O}(h) .
$$

If we neglect the Taylor remainder and use the initial boundary condition $\left(u_{s}^{\prime}\right)_{N}=-\left(1+(s-1) / 2 r_{N}\right)\left(u_{s}\right)_{N}$, this finally leads us to the relation

$$
\begin{aligned}
\frac{2}{h^{2}}\left(\dot{u}_{s}\right)_{N-1}-\left[1+\frac{2}{h^{2}}+\left(1+\frac{s-1}{2 r_{N}}\right)\left(\frac{2}{h}+\frac{s-1}{r_{N}}\right)-\left(v_{s}\right)_{N}\right] & \left(\dot{u}_{s}\right)_{N}+\left(u_{s}\right)_{N}\left(\dot{v}_{s}\right)_{N} \\
& =\frac{1}{r_{N}}\left[1+\frac{1}{h}+\frac{s-1}{r_{N}}\right]\left(u_{s}\right)_{N} .
\end{aligned}
$$

With the same considerations made on $v$, we also get

$$
\begin{aligned}
\frac{2}{h^{2}}\left(\dot{v}_{s}\right)_{N-1}-\left[\rho+\frac{2}{h^{2}}+\left(\sqrt{\rho}+\frac{s-1}{2 r_{N}}\right)\left(\frac{2}{h}+\frac{s-1}{r_{N}}\right)\right]\left(\dot{v}_{s}\right)_{N} & +\left(u_{s}\right)_{N}\left(\dot{u}_{s}\right)_{N} \\
& =\frac{1}{r_{N}}\left[\sqrt{\rho}+\frac{1}{h}+\frac{s-1}{r_{N}}\right]\left(v_{s}\right)_{N} .
\end{aligned}
$$

If we set $\dot{U}_{s}=\left(\left(\dot{u}_{s}\right)_{0}, \ldots,\left(\dot{u}_{s}\right)_{N},\left(\dot{u}_{s}\right)_{0}, \ldots,\left(\dot{u}_{s}\right)_{N}\right) \in \mathbb{R}^{2(N+1)}$, then the matrix is a $2 \times 2$ block, where each block turns out to be a tridiagonal matrix $(N+1) \times(N+1)$. In our computations, we have preferred to deal with the vector $\dot{U}_{s}=\left(\left(\dot{u}_{s}\right)_{0},\left(\dot{v}_{s}\right)_{0}, \ldots,\left(\dot{u}_{s}\right)_{N}, \ldots,\left(\dot{v}_{s}\right)_{N}\right)$ since the profile of the matrix will not depend on the number of discretization points, contrary to the first way of storing the unknowns of the problem.

Concerning continuation algorithm with respect to other parameters such as $\rho$ or $m$ in the two-dimensional case, the way of discretizing linear elliptic problems is similar.

Acknowledgements. The author would like to thank the referees for fruitful suggestions that enabled various improvements in the revised form of the manuscript.

\section{REFERENCES}

[1] M. Balabane, J. Dolbeault and H. Ounaies, Nodal solutions for a sublinear elliptic equation. Nonlinear Anal. 52 (2003) 219-237.

[2] A.V. Buryak, V.V. Steblina and Y. Kivshar, Self-trapping of light beams and parametric solitons in diffractive quadratic media. Phys. Rev. A 52 (1995) 1670-1674.

[3] A.V. Buryak, P. Di Trapani, D.V. Skryabin and S. Trillo, Optical solitons due to quadratic nonlinearities: from basic physics to futuristic applications. Phys. Rep. 370 (2002) 62-235.

[4] L. Di Menza, Transparent and absorbing conditions for the Schrödinger equation in a bounded domain. Numer. Funct. Anal. Optim. 18 (1997) 759-775.

[5] G. Fibich, N. Gavish and X.-P. Wang, Singular ring solutions of critical and supercritical nonlinear Schrödinger equations. Physica D 18 (2007) 55-86.

[6] W.J. Firth and D.V. Skryabin, Optical solitons carrying orbital angular momentum. Phys. Rev. Lett. 79 (1997) $2450-2453$.

[7] H. He, M.J. Werner and P.D. Drummond, Simultaneous solitary-wave solutions in a nonlinear parametric waveguide. Phys. Rev. E 54 (1996) 896-911.

[8] J. Iaia and H. Warchall, Nonradial solutions of a semilinear elliptic equation in two dimensions. J. Diff. Equ. 119 (1995) $533-558$.

[9] R. Kajikiya, Norm estimates for radially symmetric solutions of semilinear elliptic equations. Trans. Amer. Math. Soc. 347 (1995) 1163-1199.

[10] M.K. Kwong, Uniqueness of positive solutions of $\Delta u-u+u^{p}=0$ in $\mathbb{R}^{n}$. Arch. Rat. Mech. Anal. 105 (1989) $243-266$.

[11] D.J.B. Lloyd and A.R. Champneys, Efficient numerical continuation and stability analysis of spatiotemporal quadratic optical solitons. SIAM J. Sci. Comput. 27 (2005) 759-773.

[12] B. Malomed, P. Drummond, H. He, A. Berntson, D. Anderson and M. Lisak, Spatiotemporal solitons in multidimensional optical media with a quadratic nonlinearity. Phys. Rev. E 56 (1997) 4725-4735. 
[13] K. McLeod, W.C. Troy and F.B. Weissler, Radial solutions of $\Delta u+f(u)=0$ with prescribed number of zeros. J. Diff. Equ. 83 (1990) 368-378.

[14] T. Mizumachi, Vortex solitons for 2D focusing nonlinear Schrödinger equation. Diff. Int. Equ. 18 (2005) 431-450.

[15] I.M. Moroz, R. Penrose and P. Tod, Spherically-symmetric solutions of the Schrödinger-Newton equation. Class. Quant. Grav. 15 (1998) 2733-2742.

[16] V.V. Steblina, Y. Kivshar, M. Lisak and B.A. Malomed, Self-guided beams in diffractive $\chi^{(2)}$ medium: variational approach. Optics Comm. 118 (1995) 345-352.

[17] P.L. Sulem and C. Sulem, The nonlinear Schrödinger equation, Self-focusing and wave collapse. AMS, Springer-Verlag (1999).

[18] I.N. Towers, B.A. Malomed and F.W. Wise, Light bullets in quadratic media with normal dispersion at the second harmonic. Phys. Rev. Lett. 90 (2003) 123902.

[19] M.I. Weinstein, Nonlinear Schrödinger equations and sharp interpolation estimates. Comm. Math. Phys. 87 (1983) $567-576$. 